\title{
Report
}

\section{Mechanical Behavior of the Shielded HL-LHC Beam Screen during a Magnet Quench}

\author{
M. Morrone, C. Garion, P. Chiggiato, O. Sacristan de Frutos, M. Guinchard, L. Fiscarelli \\ CERN, Esplanade des Particules, 1211 Geneva 23, Switzerland
}

Keywords: HL-LHC, LHC, magnet quench test, mechanical measurements, shielded beam screen

\begin{abstract}
In the framework of the High-Luminosity Large Hadron Collider (HL-LHC) project, larger and shielded beam screens will be installed in the beam pipe of the superconducting magnets of the insertion next to the ATLAS and CMS detectors. The beam screen is a complex assembly that guarantees the vacuum requirements while shielding the magnet cold mass from the induced heat loads. Such an assembly has to withstand high intensity electromagnetic forces induced during magnet quenches. The mechanical stability was verified in a dedicated quench test campaign performed at the magnet test station of CERN. Strain gauges, custom-made probes, and optical fibres were installed in the beam screen to measure deformations during a quench. Magnetic field measurements were also carried out by means of pick-up coils mounted within the assembly. The main outcomes of this work are the elastic behaviour of the beam screen after 52 quenches and a satisfactory agreement between measurements and simulations.
\end{abstract}

Geneva, Switzerland

June, 2021 


\title{
Mechanical Behavior of the Shielded HL-LHC Beam Screen during a Magnet Quench
}

\author{
M. Morrone, C. Garion, P. Chiggiato, O. Sacristan de Frutos, M. Guinchard, L. Fiscarelli
}

\begin{abstract}
In the framework of the High-Luminosity Large Hadron Collider (HL-LHC) project, larger and shielded beam screens will be installed in the beam pipe of the superconducting magnets of the insertion next to the ATLAS and CMS detectors. The beam screen is a complex assembly that guarantees the vacuum requirements while shielding the magnet cold mass from the induced heat loads. Such an assembly has to withstand high intensity electromagnetic forces induced during magnet quenches. The mechanical stability was verified in a dedicated quench test campaign performed at the magnet test station of CERN. Strain gauges, custom-made probes, and optical fibres were installed in the beam screen to measure deformations during a quench. Magnetic field measurements were also carried out by means of pick-up coils mounted within the assembly. The main outcomes of this work are the elastic behaviour of the beam screen after 52 quenches and a satisfactory agreement between measurements and simulations.
\end{abstract}

Index Terms - HL-LHC, LHC, magnet quench test, mechanical measurements, shielded beam screen.

\section{INTRODUCTION}

$\mathbf{T}$ HE proposed HL-LHC shielded beam screen is an octagonally-shaped pipe that is placed within the cold bore of the new superconducting magnets, called MQXF, installed in the collider insertion regions around the ATLAS and CMS experiments [1], [2]. The beam screen protects the superconducting magnets by intercepting the collision debris and minimizing the beam impedance contributions (both resistive-wall and geometric) [3], electron cloud [4], and synchrotron radiation with a higher temperature than $1.9 \mathrm{~K}$. To ensure vacuum stability, the beam screen is perforated to allow the beam induced particles to reach the cryopumping surfaces of the magnet bore at $1.9 \mathrm{~K}$.

The mechanical integrity of the HL-LHC beam screen could be undermined by high electromagnetic forces induced by the fast decay of the magnetic field during a magnet quench, i.e., a resistive transition of the superconducting magnet. The shielded beam screen must withstand at least 50 quenches at high field and 20 years of operation [5]. To verify that the proposed design fits such requirements, a series of quench tests have been conducted at CERN.

The mechanical and thermal performance of the LHC beam screens was extensively measured during the realization phase of the LHC.

The first tests were carried out on a $1 \mathrm{~m}$ long square cross-section beam screen, tested in a short model dipole magnet [6]. Such a test was performed using four strain gauges mounted on full-bridge mode, a strain gauge mounted on a free plate to compensate any spurious effect, two carbon resistors to measure the temperature, and two mechanical deflect-meters to measure the actual deformation of the screen. The highest sampling frequency was $200 \mathrm{~Hz}$ which is too low to detect the peaks of the measurement during a quench. The deformations induced by the quench were calculated correlating the signals of the pre-calibrated strain gauges. However, the calibration was done by applying a concentrated force, though the induced forces are volumetric; such an approximation resulted in an unknown accuracy.

On a subsequent step, an $11 \mathrm{~m}$ long square cross-section beam screen was installed in an early design LHC dipole magnet for a general assembly test to measure angular and linear deformations with respect to the magnet after a few quench tests [7]. Another test was performed on a $1.15 \mathrm{~m}$ long LHC's racetrack-type beam screen inserted in a short dipole magnet [8]. The instrumentation consisted of four identical displacement transducers and two temperature gauges. The transducers were based on strain gauges that were applied on an elastic frame structure. Only the structure of two transducers was in contact with the internal side of the beam screen as the other two were used as compensators. The main output of this test was a linear relation between $B \dot{B}$, i.e., the magnetic

Manuscript receipt and acceptance dates will be inserted here.

Research supported by the HL-LHC project.

Corresponding author: Marco Morrone

M. Morrone, C. Garion, P. Chiggiato, O. Sacristan de Frutos, M. Guinchard, L. Fiscarelli are with CERN, Esplanade des Particules, 1211 Geneva 23, Switzerland. 
flux density times its time derivative, and the deformations of the beam screen. In turn, this relation was used to describe the behavior of the beam screen undergoing any magnet quench. The sampling frequency was given by a $200 \mathrm{~Hz}$ Butterworth filter, which limited the analysis to a narrow frequency range. Such a test did not show any plastic deformations even for loads twice higher than those ascribed to an LHC main dipole quench. A strong bending oscillation was found and the cause was not fully understood. It was probably due to a step-like evolution in the current decay.

Finally, a full-length LHC standard racetrack type beam screen was tested in a $15 \mathrm{~m}$ long dipole magnet which was quenched from $3 \mathrm{kA}$ to $13 \mathrm{kA}$ [9]. In addition to the sensors used in the first tests, two optical displacement transducers were developed to have a direct measurement of the beam screen deformations. A $5 \mathrm{kHz}$ acquisition frequency was adopted for the optical analysis enabling a more precise measurement. The transducers based on strain gauges gave similar results than those of the short model magnet. The beam screen was pulled against the cold bore for currents higher than $9 \mathrm{kA}$. However, the contact between the cold bore and the beam screen was affected by the screws holding the transducers onto the external side of the screen. A torsional transducer based on strain gauges was also installed. It consisted of two concentric tubes connected by thin plates whose deformation was directly related to the angular displacements. The highest measured angular displacement was $0.4^{\circ}$ but it was caused by the deformation of the cold mass during a quench rather than the beam screen itself. No permanent plastic deformation was observed at the end of the test.

This article is organized as follows. In Section II, the shielded HL-LHC beam screen is presented and the function of each component of the assembly is explained. In Section III, the magnet quench campaign, the magnet protection systems, and the equivalent beam screen used for the test are described. In Section IV, the instrumentation mounted on the beam screen to capture the expected deformations is illustrated. In Section V, a general overview of all the results of the quench campaign is given with a particular focus on the measurements at 8.2 and 18.2 kA. Finally, in Section VI the conclusions of the work are presented.

\section{THE HL-LHC SHIELDED BEAM SCREEN}

The HL-LHC beam screen, shown in Fig. 1, is made of $1 \mathrm{~mm}$ thick high-nitrogen, high-manganese stainless steel [10]. The inner surface is colaminated with $75 \mu \mathrm{m}$ thick high-purity copper to lower the beam impedance, i.e., a disturbing electromagnetic interaction leading to instabilities for high beam intensities. Amorphous carbon is deposited on the inner copper layer of the beam screen to lower its secondary electron yield and avoid multipacting and electron-cloud phenomena [11].

On the longitudinal flat surfaces of the beam screen, four series of tungsten alloy blocks and four cooling tubes are alternately placed. The blocks, also referred to as heat absorbers, intercept the collision debris; they are laid on the octagonal pipe to avoid residual stress during cooldown [12], [13]. The blocks are centered by means of pins welded on the octagonal pipe and kept in place by titanium elastic rings. The temperature of the beam screen is expected to be between $60 \mathrm{~K}$ and $80 \mathrm{~K}$, whereas the cold bore is maintained at $1.9 \mathrm{~K}$. The heat load is therefore intercepted by the heat absorbers at a temperature higher than that of the cold bore and transferred to the cooling tubes through small copper connections, the so-called thermal links. In this way, the power required by the cryogenic system is considerably reduced. The assembly is supported by titanium springs and ceramic balls within the cold bore. The nominal gap between the cold bore and the heat absorbers is $1.5 \mathrm{~mm}$. Gas cryopumping on the coldest surface is assured by slots punched in the beam screen sheet, where the cooling tubes are welded. Flexible copper-beryllium sheets are clipped onto the cooling tubes to mask the pumping holes, and therefore, intercept electrons and photons that would otherwise impinge on the cold bore leading to an increase of heat load [14]. 


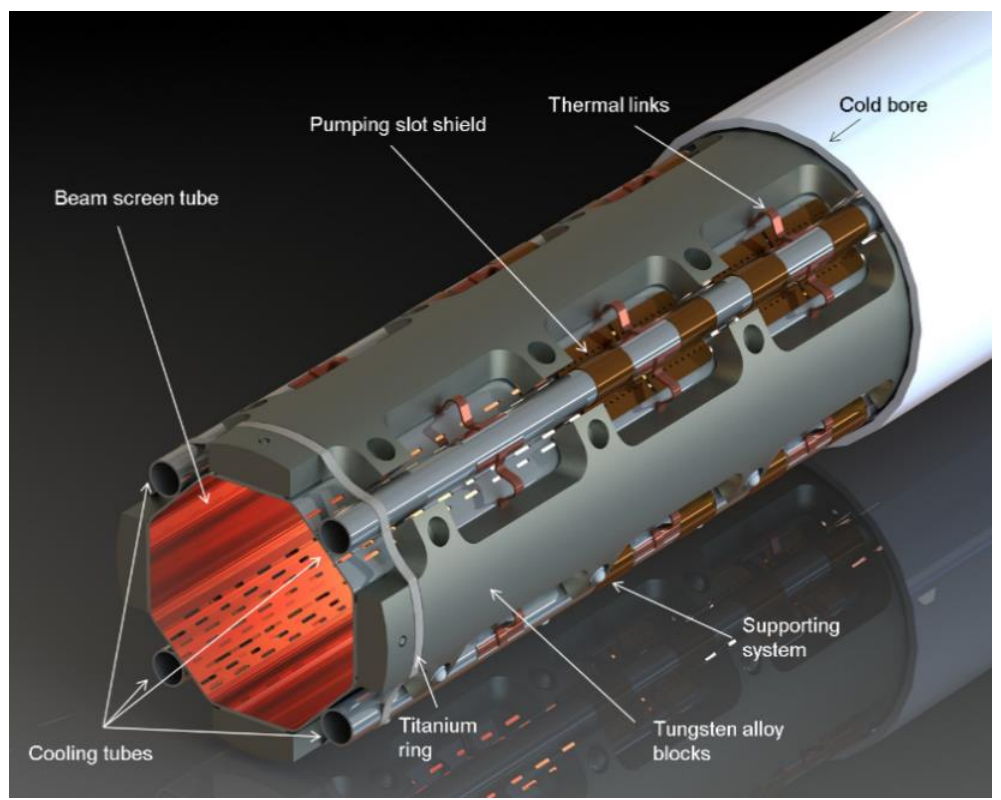

Fig. 1 HL-LHC beam screen (Q1-type).

The design of the HL-LHC beam screen is based on a complex multiphysics finite element method (FEM) approach. It includes a solving method that fully couples magnetic, thermal, and mechanical equations in a three-dimensional space [15], [16]. Previous models were based on analytical formulations in only two dimensions. The earlier method gave rise to conservative approximations and unduly limited new design solutions [17], [18]

\section{TEST CAMPAIGN}

he design of the HL-LHC beam screen needs to be experimentally validated. For this purpose, a test campaign to measure the deformations of the beam screen during a magnet quench was conducted between the 10th and the 18th of October 2018 at the magnet test station of CERN. The tested beam screen was the Q1-type, i.e., the one inserted in the MQXF quadrupole magnet denoted as Q1. The Q1 quadrupole is the closest to the collision point and the relative Q1-type beam screen has $16 \mathrm{~mm}$ thick shielding material, the thickest amongst the new shielded beam screens. The magnet used for the tests was the short model called MQXFS-4b; 52 quenches were performed in total.

The following tests have been carried out during this campaign:

- $\quad$ training verification of the MQXFS-4b at $1.9 \mathrm{~K}$;

- $\quad$ flux jump effects during test ramps and dedicated exponential cycles;

- $\quad$ magnet protection studies;

- measurement of the magnetic field during a quench by means of dedicated pick-up coils installed in the beam screen.

The Q1-type beam screen adopted for the test is shown in Fig. 2. 


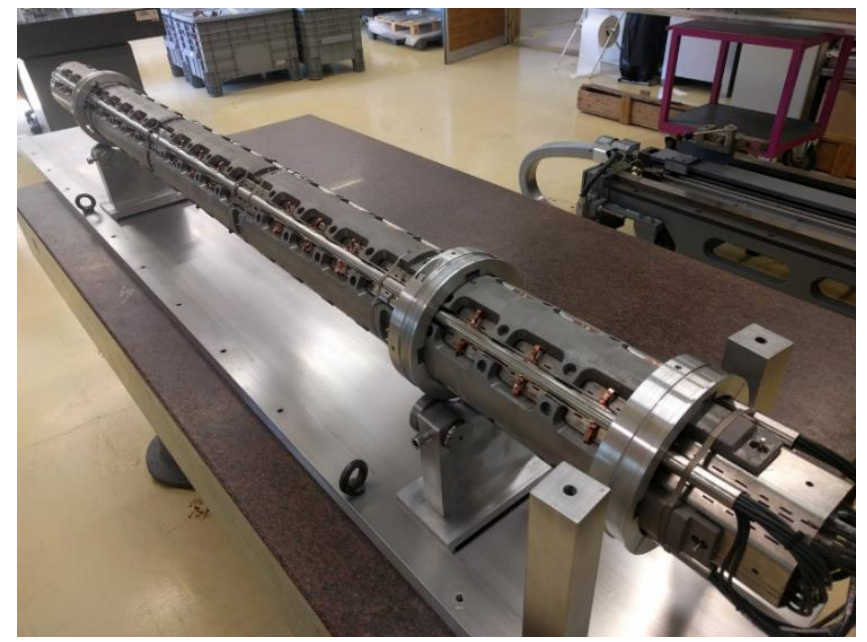

Fig. 2 HL-LHC beam screen (Q1-type) used for the quench test. The length is $2 \mathrm{~m}$ and the weight is around $100 \mathrm{~kg}$.

The beam screen was inserted horizontally into the MQXFS-4b after being secured for translational and rotational movements by dedicated flanges (see Fig. 3).

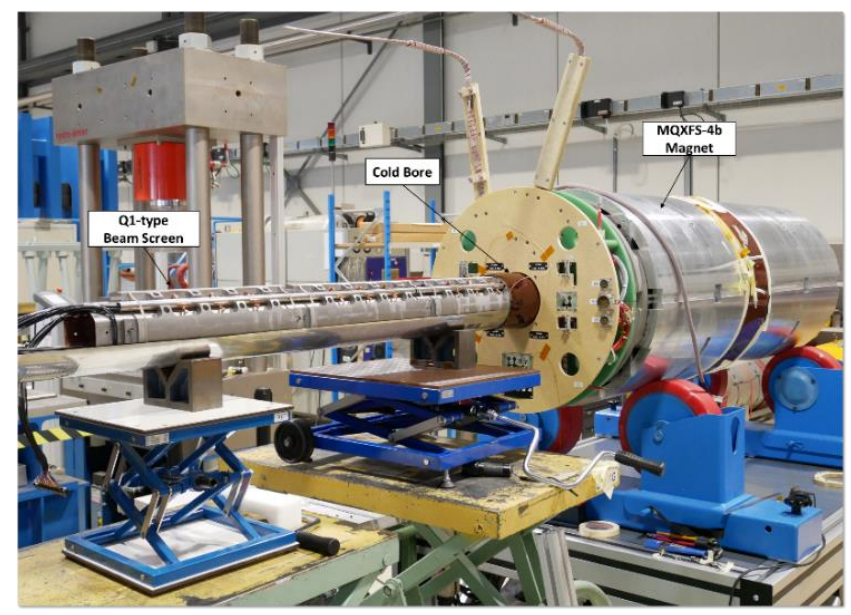

Fig. 3 HL-LHC beam screen (Q1-type) inserted in the MQXFS-4b magnet

The test was conducted in the vertical cluster station $\mathrm{G}$ at the CERN magnet test facility.

\section{A. Magnet protection system}

The distribution of Lorentz forces induced in the beam screen during a quench is closely related to the magnet protection system. The quench heaters (QHs) and the coupling-loss induced quench (CLIQ) system are active methods to protect the magnet. As the energy cannot be extracted out of the magnet it has to be released within it in the largest possible volume to increase the average temperature while avoiding local hotspot and damage. The QHs are strips made of stainless steel, partially plated with copper, and embedded in two polymide foils. The QHs are attached to the coil and connected to capacitor banks. Once the quench is detected the heaters are fired and ohmic losses are transferred uniformly to the coil by thermal diffusion. The CLIQ system relies on the generation of coupling currents between filaments and strands to uniformly spread the quench in the magnet. This is obtained by means of an external capacitor bank connected to the coil windings in a way to produce a fast change of the local magnetic field. Therefore, the heat is directly induced within the coil resulting in a much faster heating process than thermal diffusion by QHs only[19]. The baseline for the MQXF magnet is composed of the conventional QHs and the CLIQ system.

As shown in Fig. 4, the CLIQ system introduces some oscillations during the initial current discharge. Such oscillations are different for the couples of magnet poles P 1-3 and P 2-4 shown in Fig. 5. 


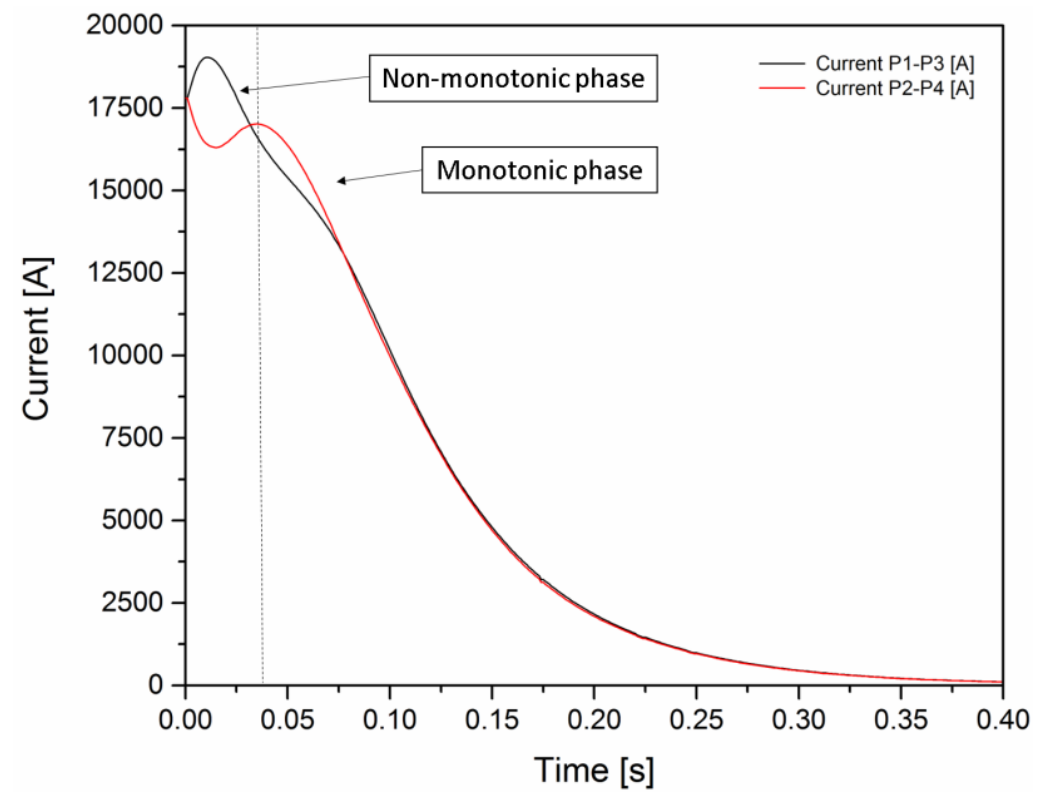

Fig. 4 Current discharge at 17.8 kA for the couple of poles P1-P3 in black and for the couple of poles P2-P4 in red. The non-monotonic and monotonic phases are separated by a dashed line in the graph.

Two phases can be identified in the transient of the current discharge as shown in Fig. 4: a non-monotonic phase in which the currents oscillate and a monotonic one where all currents decay similarly.

In the first phase, the currents increase and decrease alternatively for the couples of poles. The direction of the forces depends on the time-derivative of the magnetic flux density corresponding to the pole. Therefore, if one assumes that the current has qualitatively the same behaviour as the magnetic flux density, a local torque is induced in each region of the beam screen between two consecutive poles. The local torque is expected to change direction each time there is a variation of the current time-derivative. In the second phase, outward forces are generated. The distribution of currents and forces for the two phases is shown in Fig. 5. The net torque is zero when the beam screen is perfectly centred with respect to the poles. In case of angular or translational misalignment [20], the net torque is not zero; despite that, simulations show that the maximum allowed misalignment of $2^{\circ}$ remains acceptable [21].

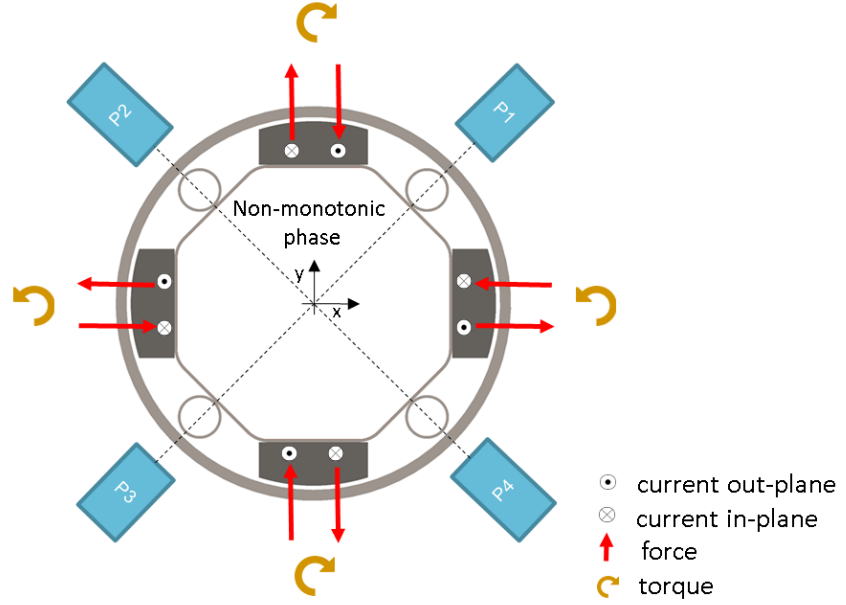

(a)

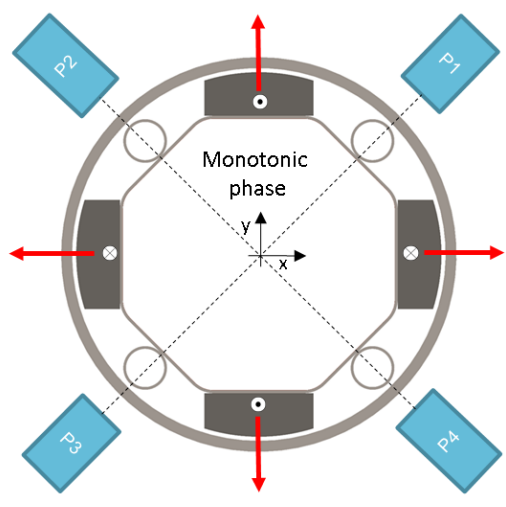

(b)

Fig. 5 Distribution of forces and currents during the non-monotonic phase in (a) and the monotonic phase in (b).

\section{B. Magnet quenches}

Several types of magnet quenches have been performed during the test, namely:

- 20 x CLIQ study (CLIQ has been used along with the QHs to protect the magnet);

- 25 x QH study (the magnet has been protected by QHs only);

- $1 \mathrm{x}$ training (the current of the magnet has been increased to a value higher than that of the previous quench threshold);

- $2 x$ extraction (the current has been extracted in an external dump); 
- $4 \mathrm{x}$ high quench integral (the quench detection system is manually delayed to investigate quench propagation and resistance build up in the magnet);

- $2 \times$ Ramp rate (the current ramp rate of the magnet has been increased to $400 \mathrm{~A} / \mathrm{s}$ instead of $1 \mathrm{~A} / \mathrm{s}$ ).

The number of quenches provoked at different current values is shown in Fig. 6.

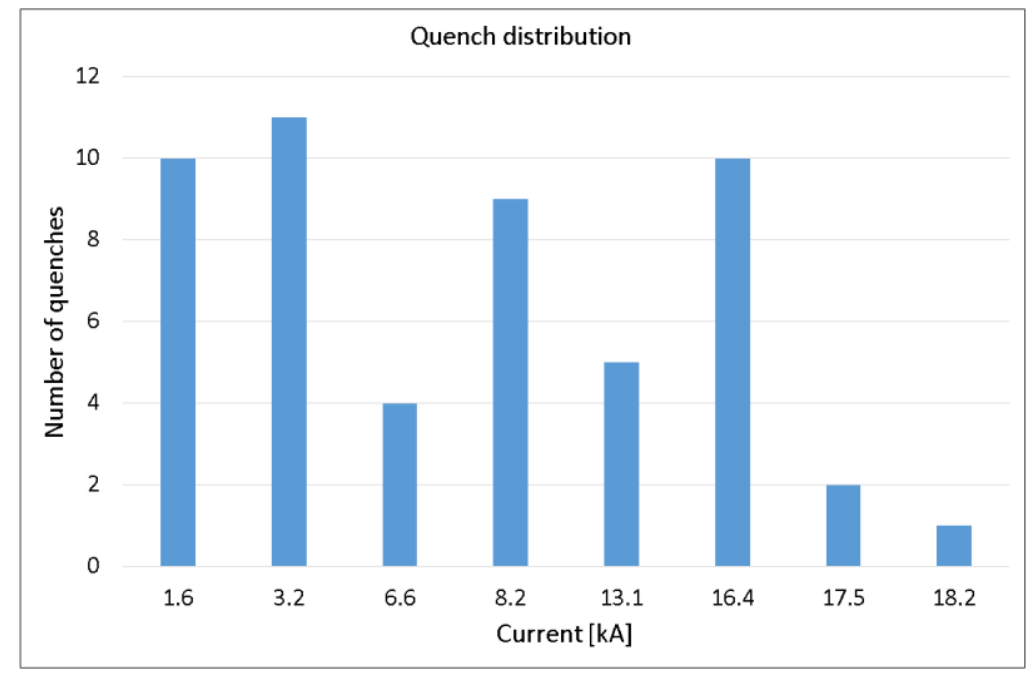

Fig. 6 Distribution of quenches at different values of current.

It is important to point out that the QH study was not indicative of real operations as the current of the magnet was discharged in an external dump resistor, which translates into an artificial but faster decay of the magnetic field. This allows to save time during the test as there is no need to cool down the magnet for subsequent quenches. Therefore, the mechanical response of the beam screen was not representative and this article will cover the CLIQ study only.

Nevertheless, the CLIQ study has been performed in the short MQXFS-4 magnet (1.192 m of magnetic length). Some modifications of the CLIQ unit were necessary to have a similar behaviour with respect to the MQXF-Q1 series magnet (4.2 m of magnetic length), in particular:

- $\quad$ To assure the same frequency of the current in the couple of poles, the CLIQ unit capacitance should be increased by a factor of 3.5. However, this was not possible as the unit has a maximum capacitance only 1.25 times higher than the baseline, and it was decided to set the capacitance to the same value as the baseline. Therefore, the oscillating frequency is roughly $3.25 \sim 1.9$ times higher. This has many implications on the transient, in particular, reducing the CLIQ performance [22];

- To attain the same peak power density, the ratio (charging voltage)/(magnet length) should be kept constant, i.e. $600 \mathrm{~V}$ $/(4.2 \mathrm{~m} / 1.192 \mathrm{~m}) \sim 170 \mathrm{~V}$. The CLIQ unit of the test station had only a limited number of charging voltage selections, i.e. every $100 \mathrm{~V}$, so for the CLIQ study it has been rounded to $100 \mathrm{~V}$ and $200 \mathrm{~V}$. Matching the peak power density is relevant at high current [22];

- To reach the same energy density, the ratio (CLIQ energy)/(magnet length) should be constant. For the same capacitance, the energy is proportional to the square of the charging voltage $\sqrt{600 \mathrm{~V}^{2} /(4.2 \mathrm{~m} / 1.192 \mathrm{~m})} \sim 320 \mathrm{~V}$. Matching the energy density is relevant at low to medium current [22].

The magnetic flux density $B$ and its time derivative $\dot{B}$ are the magnetic parameters affecting the Lorentz forces. Assuming a conservative case in which electro-dynamic effects for short time scales $(\sim 10 \mathrm{~ms})$ are not considered, we can assume that the current $I$ is proportional to $B$ and refer to $I$ for the forces induced in the beam screen.

The highest torque is induced during the first CLIQ oscillation and, therefore, it highly depends on $d I_{\text {star }} / d t$. The lower capacitance set in the CLIQ unit has no direct influence on $d I_{\text {star }} / d t$ but only on the oscillating frequency which, in turn, does not perturb the validity of the test [23]. The voltage of the CLIQ unit is proportional to $d I_{\text {star }} / d t$. Therefore, as the voltage cannot be set at the ideal $170 \mathrm{~V}, d I_{\text {star }} / d t$ was different with respect to that of the MQXF magnet. In particular, at $100 \mathrm{~V} d I_{\text {star }} / d t$ is $41 \%$ lower whereas at $200 \mathrm{~V}$ it is $17.5 \%$ higher.

\section{Test-type beam screen}

The beam screen used for the magnet quench test was modified with respect to the nominal version. 
The octagonal tube was made of AISI 316L stainless steel instead of stainless steel P506 for ease of procurement. The P506 was specially developed at CERN [24] for the low relative magnetic permeability at cryogenic temperatures. As the magnetic field quality is not relevant for the purpose of the test, the $316 \mathrm{~L}$, having the same electrical properties, has been adopted, see Table 1. Additionally, the stiffness of the two materials is similar, around $206 \mathrm{GPa}$ for the $316 \mathrm{~L}$ and $240 \mathrm{GPa}$ for the P506. At $4.2 \mathrm{~K}$, the yield and tensile strength for the 316 are $431 \mathrm{MPa}$ and $1441 \mathrm{MPa}$ [25], respectively, whereas for the P506 these values are 1620 $\mathrm{MPa}$ and $2115 \mathrm{MPa}$ [24], respectively. However, due to the conditions of the test, other modifications were needed. In fact, the magnet was immersed in the helium bath of the cryostat at $1.9 \mathrm{~K}$ and so was the beam screen. Therefore, the temperature was different from the real temperature range in which it will operate (60-80 K). This has important consequences for the validity of the test as the electrical resistivity of some beam materials changes significantly from $1.9 \mathrm{~K}$ to $60 \mathrm{~K}$ (see Table 1 ).

\begin{tabular}{ccc}
\multicolumn{2}{c}{ Table 1 Electrical resistivity of the beam screen materials at $1.9 \mathrm{~K}$ and $60 \mathrm{~K}}$. \\
\hline \hline Material & $\begin{array}{c}\text { Electrical resistivity } \\
\text { @ } 1.9 \mathrm{~K}[\Omega \cdot \mathrm{m}]\end{array}$ & $\begin{array}{c}\text { Electrical resistivity } \\
@ 60 \mathrm{~K}[\Omega \cdot \mathrm{m}]\end{array}$ \\
\hline Copper OFE [26] & $1.56 \cdot 10^{-10}$ & $1.08 \cdot 10^{-9}$ \\
P506 - 316 L [27] & $5.52 \cdot 10^{-7}$ & $5.85 \cdot 10^{-7}$ \\
Tungsten alloy [28] & $2.87 \cdot 10^{-8}$ & $3.72 \cdot 10^{-8}$ \\
\hline \hline
\end{tabular}

For copper the electrical resistivity at $1.9 \mathrm{~K}$ is lower than the one at $60 \mathrm{~K}$ by almost a factor 7 . For stainless steel grades (P506 and $316 \mathrm{~L}$ ) and the tungsten alloy the difference is negligible. The electrical resistivity of copper is around 2 and 3 orders of magnitude lower than the one of the other materials. Therefore, copper components play a dominant role during a magnet quench as the induced forces depend linearly on resistivity. To have an equivalent behavior of the beam screen at the temperature of the test the copper inner layer and the thermal links should be resized.

As the total force induced during a quadrupole magnet quench in any electrically conductive object is equal to:

$$
F \propto \frac{G \dot{G} x^{3} t}{\rho} M
$$

where $G$ is the magnet gradient, $\dot{G}$ is its time derivative, $x$ is the distance of the component from the center, $t$ is the thickness of the object, $\rho$ is the electrical resistivity and $M$ is a geometrical parameter depending on the shape of the object. Fig. 7 shows the dimensional parameters $x$ and $t$.

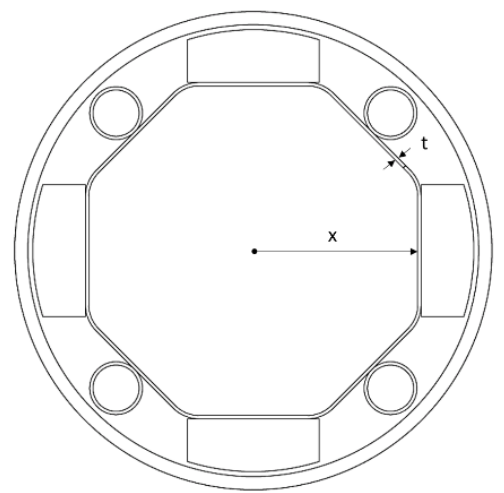

Fig. 7 The forces induced in the beam screen during a quadrupole quench depend on the distance of the component from the geometrical and magnetic centre, $x$, and its thickness, $t$.

The easiest way to have the same forces at $1.9 \mathrm{~K}$ and $60 \mathrm{~K}$ is to reduce the thickness of the inner layer so that the ratio of the thicknesses is equal to the ratio of the electrical resistivities at the two temperatures. The magnetoresistance and the ResidualResistance Ratio (RRR) of the electroplated copper layer were accounted for as well. The magnetoresistance effect has been considered for a magnetic flux density of 5T, i.e. when $|G \dot{G}|$ is at maximum. The measured RRR of the electroplated copper is 90 [29]. Therefore, the equivalent copper thickness needs to be $21 \mu \mathrm{m}$. Some measurements of the actual thickness deposited on the beam screen have been performed resulting in an average of $24.7 \mu \mathrm{m}$.

The forces induced in the other copper elements of the beam screen, i.e. the thermal links, do not follow (1).

Each absorber is $40 \mathrm{~cm}$ long and contains 3 thermal links, two at the extremity and one at the center.

The central thermal links behave as components independent from the rest of the assembly. In these links an eddy current loop is formed and the total forces read:

$$
F \propto \frac{B \dot{B} l^{2} t}{\rho} M
$$


where $B$ is the magnetic flux density, $\dot{B}$ is its time derivative and $l$ is the distance from the mid-plane of the thermal link. Fig. 8 shows the parameters $x$ and $t$.

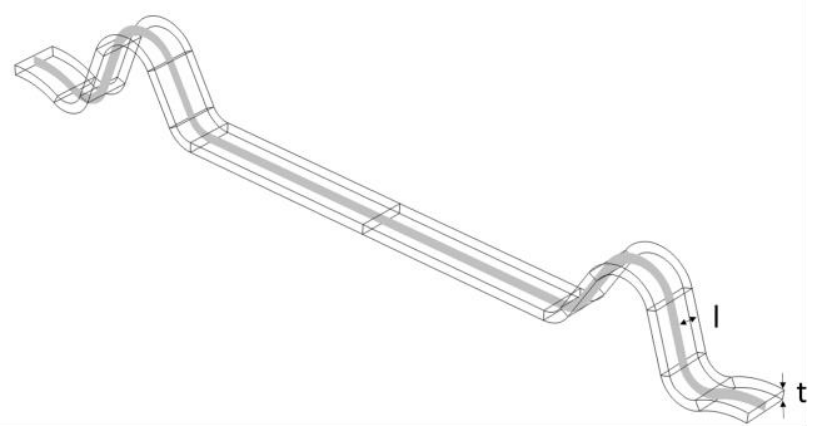

Fig. 8 The forces induced in the internal thermal link during a quench depend on the distance, $l$, from its symmetry plane (in grey in the picture), and its thickness, $t$

The thermal link is made of a solid central plate $1 \mathrm{~mm}$ thick welded to two curved segments that provide flexibility during a quench. The flexibility is obtained by stacking 10 layers of equal thickness, i.e. $0.1 \mathrm{~mm}$. Reducing the thickness of the link and so the number of layers would not be representative of the operational conditions. A more efficient solution was to reduce the width of the link as its contribution to the total forces rises to the power of 2 . It is sufficient reducing $l$ from the nominal $5 \mathrm{~mm}$ to $2.6 \mathrm{~mm}$ to obtain an equivalent force at the two temperatures. The same considerations on the magnetoresistance taken for the inner layers apply also here. The RRR of the copper thermal links is 100.

Contrary to the internal thermal links, the external ones do not behave as independent components. These act as pathways for the current induced in each quarter of the beam screen facing the poles (see Fig. 9). Thus, the current flows in two consecutive half absorbers passing through the external links to form a loop and not through the stainless steel tube as originally expected by considering the absorber fully bonded on the tube. Post-test simulations have shown that this is most likely due to the poor contact, hence high electrical resistance, between the absorbers and the tube. Therefore, the forces induced in these links depend on the current induced in the absorber rather than the dimension of the link. In turn, the current depends on the electrical resistivity of the absorber which is only slightly lower at $1.9 \mathrm{~K}$ than at $60 \mathrm{~K}$, see Table 1.

A reduction of the external links dimensions would not change significantly the current in the loop. Therefore, the nominal width of $5 \mathrm{~mm}$ was adopted for all thermal links.

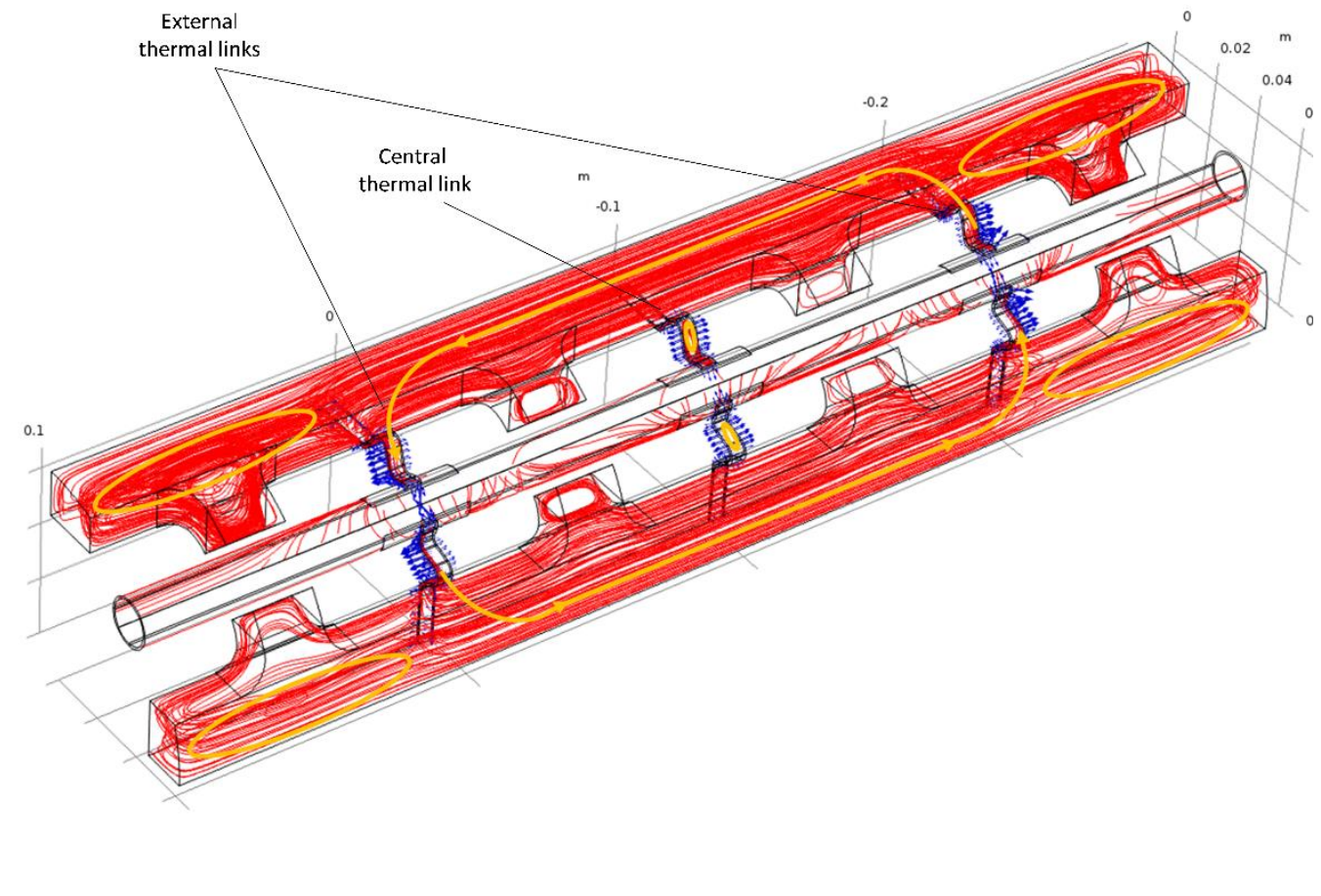


(a)

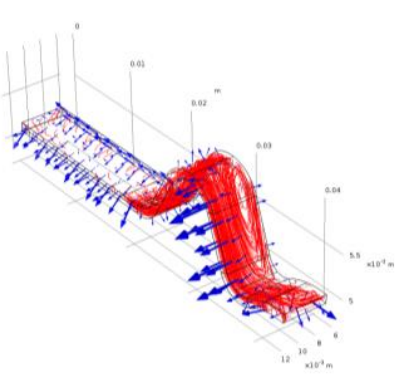

(b)

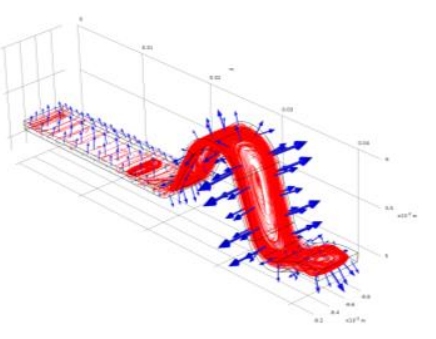

(c)

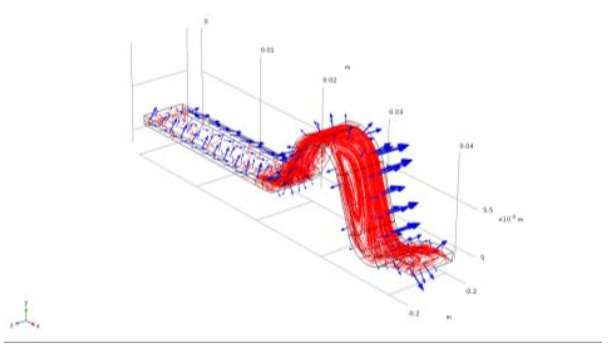

(d)

Fig. 9 Current distribution in red (simplified path in yellow) and force distribution in blue induced in a quarter of the assembly in (a), in the external-left thermal link in (b), in the central thermal link in (c), and in the external-right thermal link in (d). These current and force distributions were computed considering an high electrically insulated layer to account for the contact between the absorber and the octagonal tube.

\section{INSTRUMENTATION}

The mechanical instrumentation mounted on the beam screen consisted of the following:

- $\quad$ strain gauges to measure axial and transversal strains on 20 points distributed along three different sections of the beam screen;

- custom made probes to measure the expansion of the beam screen, the tilt of the heat absorber during the CLIQ phase and the magnetic field;

- optical fibers to measure axial and transversal strains on the outer surface of the cold bore.

All the gauges are placed in a $0.8 \mathrm{~m}$ long portion of the beam screen centred in the $1.5 \mathrm{~m}$ long magnet coil to capture the nominal magnetic field. At the extremities of this portion the magnetic field is $0.5 \%$ lower than the nominal one due to border effects.

\section{A. Strain gauges}

The strain induced in the outer surface of the beam screen was determined in the axial and transversal directions at 20 different measurement points using biaxial strain gauges.

The strain gauges XC11-3/350 used to instrument the beam screen have been provided by the company HBM. The installation of the strain gauges was performed according to [30].

Spurious effects as:

- $\quad$ thermal expansions;

- temperature-dependent variations in the strain gauge and wire resistance;

- $\quad$ thermal contractions of the measuring grid foil;

- $\quad$ magnetic fields

were deducted from the measurements by means of two compensator strain gauges. The compensators were installed on a free plate made of the same material as the beam screen and oriented in the same directions as the active strain gauges.

The strain gauges mounted on the beam screen were located in three adjacent points $(1,2,3)$ on the four faces (F1, F2, F3, F4) along three different sections, i.e. bottom (B), medium (M), and upper (U), (see Figs. 10 - 11).

The strain gauge instrumentation was read by 3 QuantumX 1615b and 3 QuantumX 840b HBM modules at $1200 \mathrm{~Hz}$.

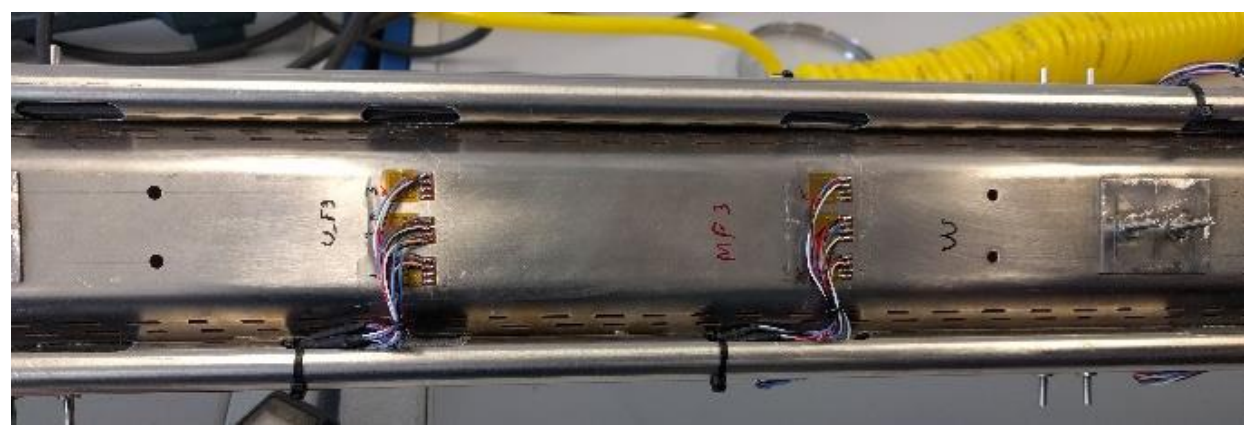

(a) 


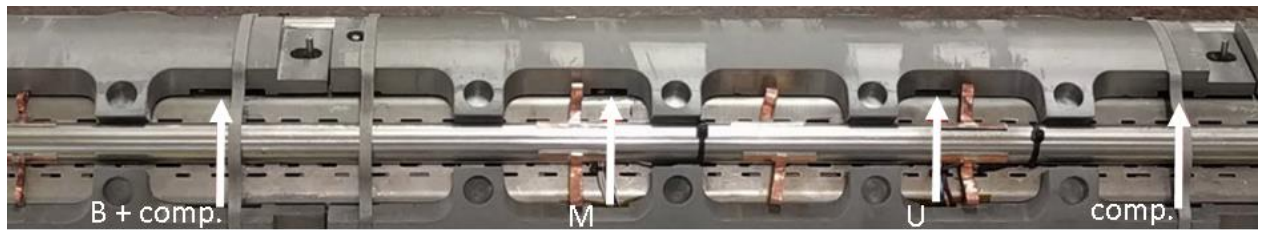

(b)

Fig. 10 In (a) strain gauges mounted on the beam screen whose cables have been confined into the cooling tubes. In (b) the dedicated slots of the tungsten block to house the strain gauges and the compensators are indicated by white arrows.

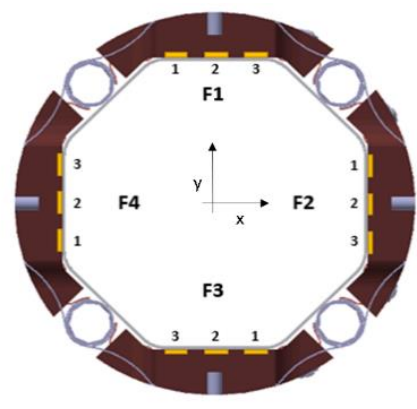

(a)

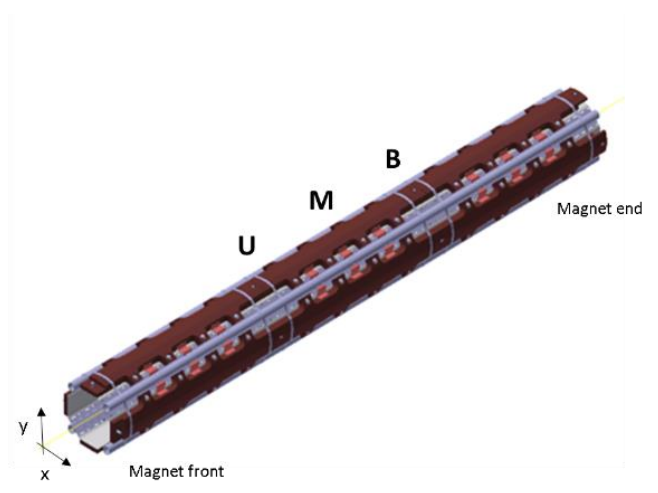

(b)

Fig. 11 Location of the strain gauges on the faces (F1, F2, F3, F4) of the beam screen in (a) and along the upper (U), middle (M), and bottom (B) section in (b).

\section{B. Custom made probes}

Three special probes were developed to measure the tilt of the absorbers and the expansion of the beam screen. Four pick-up coils have been mounted in the central probe to measure the magnetic field (see Fig. 12).

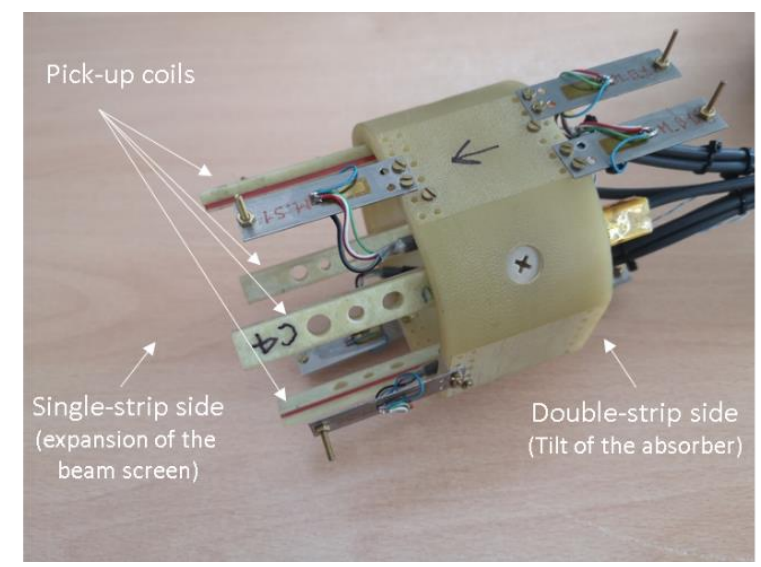

Fig. 12 Central probe where four pick-up coils have been inserted.

The probes are based on thin-cantilevered strips holding a pin at their extremity. The strains induced on the surface of the strip by a normal displacement is measured (see Fig. 13).

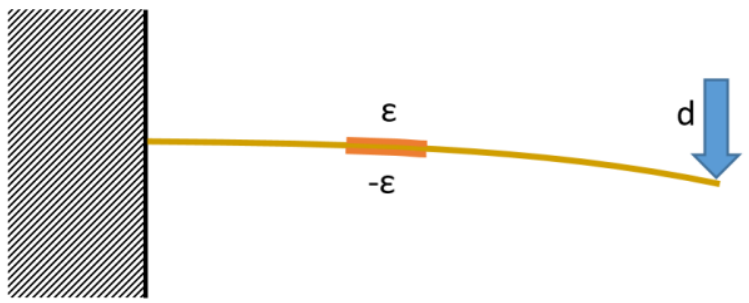

Fig. 13 Thin-cantilevered plate where the displacement, $d$, on the extremity is correlated to the measured strain, $\varepsilon$, in the strip.

The structure of the probe is made of G-11 FR-5. It is designed to be auto-equilibrated by means of elastic strips held against the beam screen and the heat absorbers (see Fig. 14). 
The tilt of the heat absorber is measured by the side of the probe where two strips are placed next to each other. The expansion of the beam screen is measured by the side of the probe containing single strips.

Each probe requires eight strain gauges, four for each side of the probe. These were calibrated against imposed deformation according to [31].

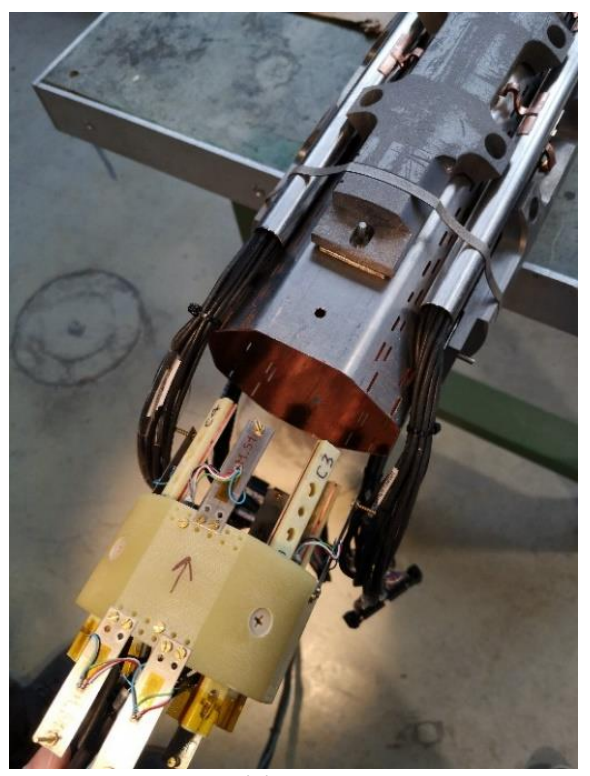

(a)

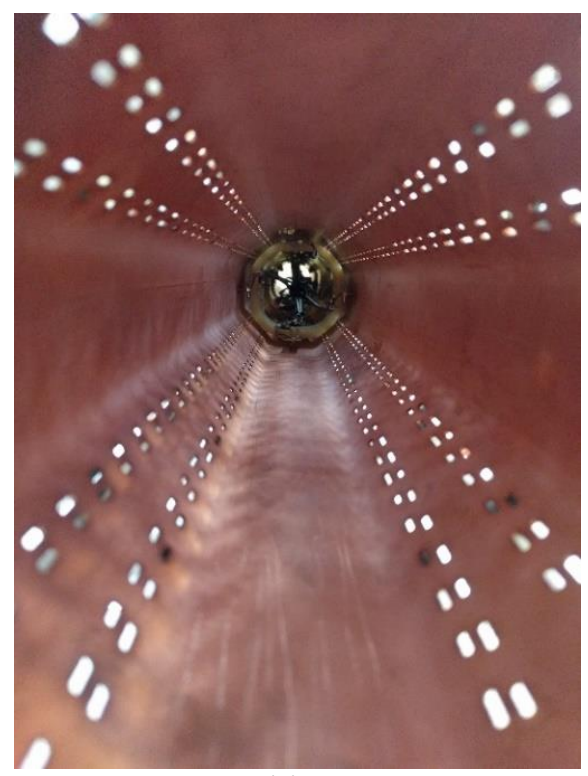

(b)

Fig. 14 In (a) insertion of the probe within the beam screen, in (b) probe in its auto-equilibrated position.

The strips were manufactured in titanium grade 5 to minimize the magnetic forces and the spurious deformations induced during a quench. To this purpose, some optimization was performed by simulation.

The pins of the double-strip-side of the probe are in contact with the absorber (see Fig. 15). Some holes are present in the beam screen to enable such a contact (see Fig. 10a). Assuming that the probe does not move during the measurement, the local tilt is calculated as the relative difference of each pair of sensors. This assumption is reasonable for short time scales due to the inertia of the probe (around 5-10 ms, non-monotonic phase of CLIQ). The pins are placed at a distance of $22 \mathrm{~mm}$ and the tilt angle is hence calculated as:

$$
\alpha=\arctan \left(\frac{t i l t}{22}\right)
$$

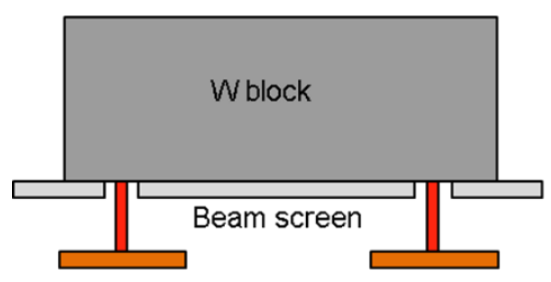

NOMINAL CONFIGURATION

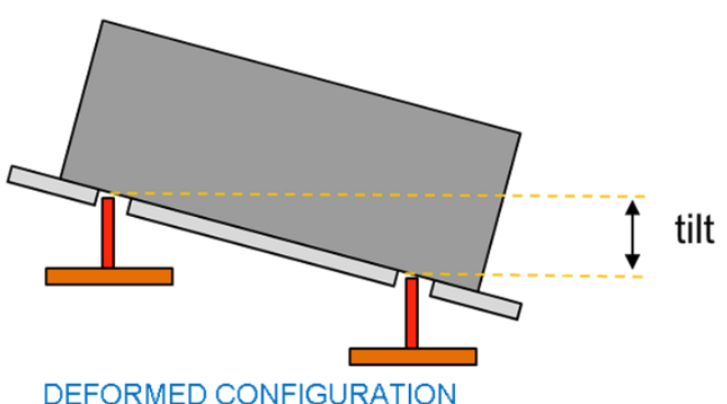

DEFORMED CONFIGURATION

Fig. 15 The tilt of the heat absorber is measured as the difference between the left and the right strips of the deformed configuration.

The pins of the single-strip-side of the probe are in contact with the inner side of the beam screen (see Fig. 16). The difference between the deformed and the nominal configurations measured by one strip is half the beam screen expansion. The total expansion, considering the force distribution shown in Fig. 5 (b), is equal to the deformation measured by two opposite strips. 


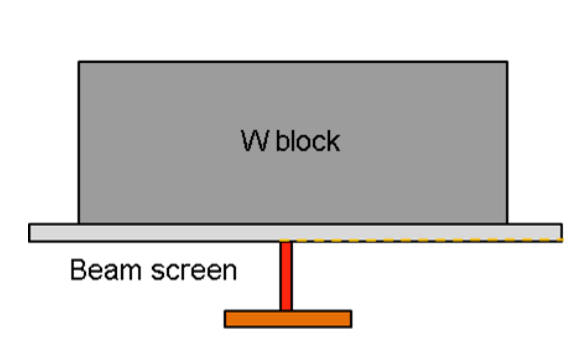

NOMINAL CONFIGURATION

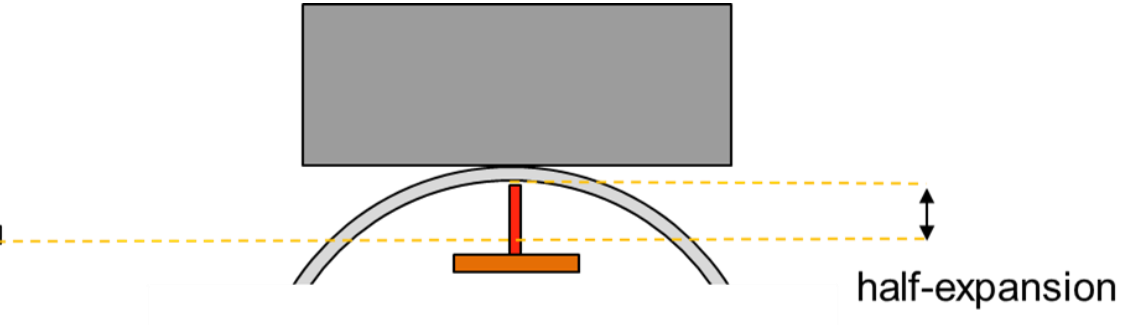

DEFORMED CONFIGURATION

Fig. 16 The expansion of the beam screen is measured from the difference between the deformed and the nominal configurations.

Two strain gauges compensators have been installed on two free strips of the upper probe.

The pick-up coils used for the measurement of the magnetic field are based on induction coils mounted in a fixed position [32], [33]. These were installed in the probe at incrementing angles of $45^{\circ}$ degrees to face the magnet poles and hence to maximize the sensitivity. The field is measured at a radial distance of $34.25 \mathrm{~mm}$, i.e. the distance of the coils with respect to the magnet center. The voltage signals, induced in each coil during the ramps, were acquired by a fast acquisition system triggered by the start of the discharge. The signals were then integrated off-line to get the flux. The sensitivity coefficients of the pick-up coils were calibrated "in-situ" by comparing the total flux intercepted by each coil with a measurement taken in the same magnet in a previous test. The setup allows the measurement of the quadrupolar components generated by the main current during the balanced powering as well as those given by the unbalanced powering of the CLIQ discharge. Given the symmetry of the magnet, the average of measurements from the four coils, corrected for the polarity, gives the quadrupolar field component whereas the difference of measurements from two consecutive poles gives the distortive components produced by CLIQ.

\section{Optical Fibers}

Strains on the outer surface of the cold bore were measured by means of optical fibers containing several measurement points. Still in the development phase for usage at $4 \mathrm{~K}$, optical fibers provide a convenient solution as they are insensitive to magnetic fields [34]. Four optical fibers have been installed in four diametrically opposed generatrixes of the cold bore, each one containing six gratings, at four angles of orientation (see Fig. 17).

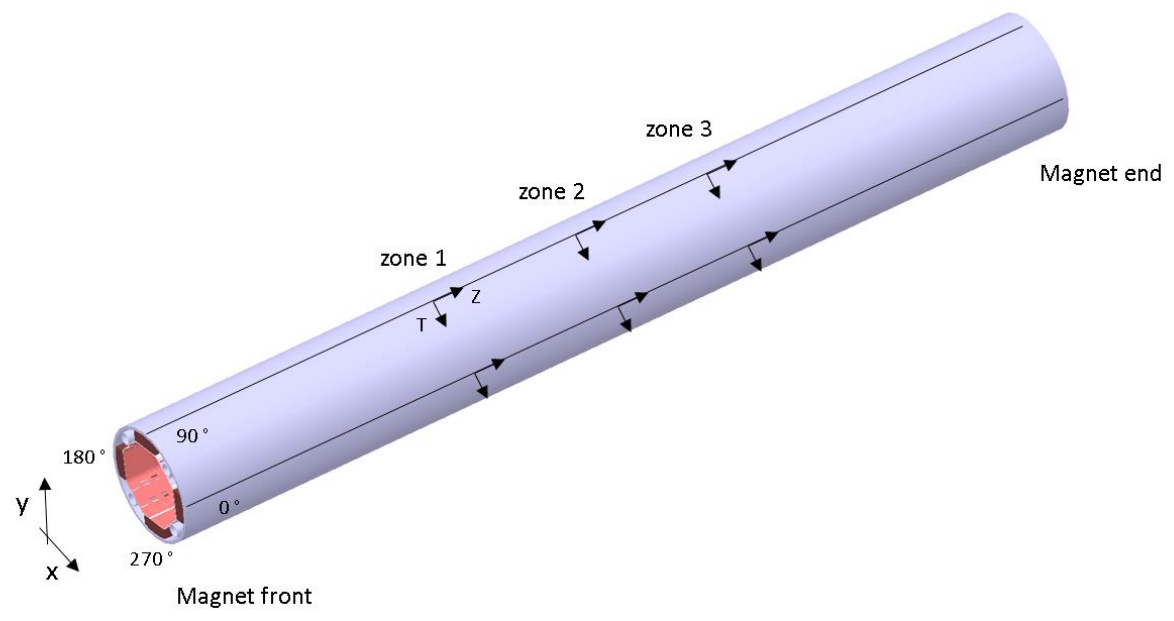

Fig. 17 Optical fibers to measure longitudinal (L) and transversal (T) strains of the cold bore mounted at $0^{\circ}, 90^{\circ}, 180^{\circ}$ and $270^{\circ}$ degree angle in three zones, i.e. zone 1 , zone 2 , and zone 3 .

Two gratings close to each other allow the measurement of transversal and axial strains in the same zone resulting in three zones of measurement per fiber (see Fig. 18). 


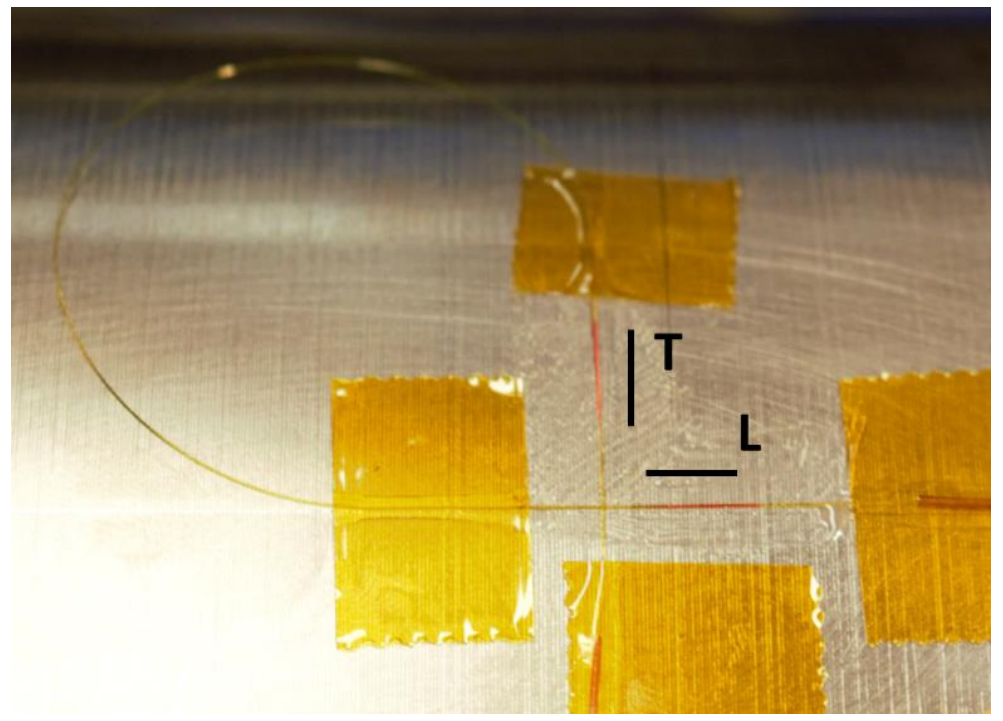

Fig. 18 Optical fiber glued as a loop to measure longitudinal (L) and transversal (T) strains in the same zone.

The optical fiber arrays were composed of $7.75 \mathrm{~m}$ polyimide fiber containing six Bragg gratings with wavelengths of 1523, 1529, $1554,1575,1581$, and $1587 \mathrm{~nm}$. The optical fibers were read by a FS22DI optical interrogator. All the instrumentation was sampled at $1200 \mathrm{~Hz}$.

\section{RESULTS AND DISCUSSION}

The list and the characteristics of the 52 quenches are shown in Table 2. The $B \dot{B}$ parameter, derived from the magnetic measurements, is a good indicator to compare the intensity of the induced forces.

In case of CLIQ, $B \dot{B}$ is reported for the first oscillation of the non-monotonic phase as well as for the highest absolute value of the monotonic phase. The difference of $B \dot{B}$ between the couple of poles 2-4 and 1-3 during the first oscillation provides an insight on the highest local torque. In the case of QHs, the highest value of the monotonic decay $|B \dot{B}|$ is provided for comparison.

For the design of the beam screen the difference of $B \dot{B}$, taken into account for the first CLIQ oscillation between two nonconsecutive poles, is $48 \mathrm{~T}^{2} / \mathrm{s}$, whereas $|B \dot{B}|$ for the monotonic decay is $165 \mathrm{~T}^{2} / \mathrm{s}$, both evaluated at the same radial distance of the measurements, i.e. $34.25 \mathrm{~mm}$.

Table 2 Technical characteristics of each magnet quench. Cells of the same colour highlight the same type of test. 


\begin{tabular}{|c|c|c|c|c|c|c|c|c|c|c|c|}
\hline \multirow{2}{*}{$\begin{array}{l}\text { File name } \\
\text { ID }\end{array}$} & \multirow{2}{*}{$\begin{array}{l}\text { Test } \\
\text { Type }\end{array}$} & \multirow{2}{*}{$\begin{array}{c}\text { Current } \\
{[\mathrm{A}]}\end{array}$} & \multirow{2}{*}{$\begin{array}{c}\text { Max } \\
\text { Gradient }[\mathrm{T} / \mathrm{m}]\end{array}$} & \multirow{2}{*}{$\begin{array}{c}\mathrm{V}_{-} \mathrm{CLIQ} \\
{[\mathrm{V}]}\end{array}$} & \multirow{2}{*}{$\begin{array}{c}\text { C_CLIQ } \\
{[\mathrm{mF}]}\end{array}$} & \multicolumn{2}{|c|}{ B_dot [T/s] wrt 1st CLIQ oscillation } & \multicolumn{3}{|c|}{ B B_dot $\left[\mathrm{T}^{2} / \mathrm{s}\right]$ wrt 1st CLIQ oscillation } & \multirow{2}{*}{$\frac{\text { Max abs (B B_dot) }\left[\mathrm{T}^{2} / \mathrm{s}\right]}{\mathrm{P}(1-2-3-4)}$} \\
\hline & & & & & & $\mathbf{P ( 1 - 3 )}$ & $P(2-4)$ & $P(1-3)$ & $P(2-4)$ & $P(1-3)-P(2-4)$ & \\
\hline tb017 & Extraction & 3298 & 31.30 & - & - & - & - & - & - & - & - \\
\hline $\mathrm{a} 001$ & CLIQ study & 8235 & 69.34 & 100 & 40 & 2.82 & -2.91 & 7.05 & -7.27 & 14.32 & 7.70 \\
\hline $\mathrm{a} 002$ & CLIQ study & 13172 & 107.38 & 100 & 40 & 2.65 & -2.79 & 10.40 & -10.80 & 21.20 & 45.40 \\
\hline $\mathrm{a} 003$ & CLIQ study & -1 & 5.87 & 200 & 40 & - & - & - & - & - & - \\
\hline $\mathrm{a} 004$ & CLIQ study & 1648 & 18.58 & 200 & 40 & 3.94 & -4.01 & 2.00 & -1.98 & 3.98 & 0.06 \\
\hline $\mathrm{a} 005$ & CLIQ study & 3297 & 31.29 & 200 & 40 & 5.14 & -5.02 & 5.00 & -4.80 & 9.80 & 0.30 \\
\hline $\mathrm{a} 006$ & CLIQ study & 8234 & 69.33 & 200 & 40 & 5.70 & -5.85 & 14.20 & -14.60 & 28.80 & 7.70 \\
\hline $\mathrm{a} 007$ & CLIQ study & 13171 & 107.38 & 200 & 40 & 5.43 & -5.68 & 21.06 & -22.00 & 43.06 & 48.50 \\
\hline $\mathrm{a} 008$ & Training & 18223 & 146.31 & 200 & 40 & 5.41 & -6.00 & 28.33 & -31.58 & 59.91 & 153.00 \\
\hline $\mathrm{a} 009$ & Quench heater study & 1648 & 18.6 & - & - & - & - & - & - & - & 1.35 \\
\hline $\mathrm{a} 010$ & Quench heater study & 3296 & 31.3 & - & - & - & - & - & - & - & 5.40 \\
\hline $\mathrm{a} 011$ & Quench heater study & 8234 & 69.3 & - & - & - & - & - & - & - & 32.00 \\
\hline $\mathrm{a} 012$ & Quench heater study & 1646 & 18.6 & - & - & - & - & - & - & - & 1.33 \\
\hline $\mathrm{a} 013$ & Quench heater study & 3295 & 31.3 & - & - & - & - & - & - & - & 5.38 \\
\hline $\mathrm{a} 014$ & Quench heater study & 8234 & 69.3 & - & - & - & - & - & - & - & 31.94 \\
\hline $\mathrm{a} 015$ & Quench heater study & 13172 & 107.4 & - & - & - & - & - & - & - & 79.35 \\
\hline $\mathrm{a} 016$ & Quench heater study & 13171 & 107.4 & - & - & - & - & - & - & - & 79.57 \\
\hline $\mathrm{a} 017$ & High quench integral & 1648 & 18.6 & - & - & - & - & - & - & - & 0.06 \\
\hline $\mathrm{a} 018$ & High quench integral & 3297 & 31.3 & - & - & - & - & - & - & - & 0.30 \\
\hline $\mathrm{a} 019$ & High quench integral & 8235 & 69.3 & - & - & - & - & - & - & - & 7.76 \\
\hline $\mathrm{a} 020$ & High quench integral & 16460 & 132.7 & 200 & 40 & 5.53 & -5.84 & 26.36 & -27.81 & 54.17 & 107.53 \\
\hline $\mathrm{a} 021$ & Ramp rate & 6070 & 52.7 & - & - & - & - & - & - & - & 2.58 \\
\hline $\mathrm{a} 022$ & Quench heater study & 16459 & 132.7 & - & - & - & - & - & - & - & 0.00 \\
\hline $\mathrm{a} 024$ & Quench heater study & 8234 & 69.3 & - & - & - & - & - & - & - & 32.26 \\
\hline $\mathrm{a} 025$ & Quench heater study & 16459 & 132.7 & - & - & - & - & - & - & - & 122.58 \\
\hline $\mathrm{a} 026$ & Extraction & 16460 & 132.7 & 100 & 40 & 2.70 & -2.88 & 12.80 & -13.70 & 26.50 & 101.61 \\
\hline $\mathrm{a} 027$ & Quench heater study & 17789 & 143.0 & 200 & 40 & 5.44 & -6.40 & 27.84 & -31.48 & 59.32 & - \\
\hline $\mathrm{a} 028$ & CLIQ study & -1 & 5.9 & - & - & - & - & - & - & - & 0.00 \\
\hline $\mathrm{a} 029$ & Quench heater study & 16459 & 132.7 & - & - & - & - & - & - & - & 120.43 \\
\hline $\mathrm{a} 030$ & Quench heater study & 16458 & 132.7 & - & - & - & - & - & - & - & 120.43 \\
\hline $\mathrm{a} 031$ & CLIQ study & 1666 & 18.7 & 200 & 50 & 3.95 & -4.14 & 2.00 & -2.04 & 4.04 & 0.06 \\
\hline $\mathrm{a} 032$ & CLIQ study & 3321 & 31.5 & 200 & 50 & 5.23 & -4.88 & 4.88 & -5.05 & 9.93 & 0.31 \\
\hline $\mathrm{a} 033$ & CLIQ study & 8277 & 69.7 & 200 & 50 & 5.68 & -5.81 & 14.23 & -14.49 & 28.72 & 7.74 \\
\hline $\mathrm{a} 034$ & CLIQ study & 1683 & 18.9 & 200 & 30 & 3.80 & -3.92 & 1.91 & -1.94 & 3.85 & 0.06 \\
\hline a035 & CLIQ study & 3320 & 31.5 & 200 & 30 & 5.15 & -4.96 & 4.97 & -4.78 & 9.75 & 0.30 \\
\hline a036 & CLIQ study & 6627 & 57.0 & 200 & 30 & 5.66 & -5.77 & 14.16 & -14.37 & 28.53 & 7.74 \\
\hline a037 & CLIQ study & 6639 & 57.0 & 200 & 20 & 5.55 & -5.66 & 13.86 & -14.17 & 28.03 & 7.74 \\
\hline $\mathrm{a} 038$ & CLIQ study & 3135 & 30.0 & 200 & 20 & 4.87 & -4.76 & 4.70 & -4.53 & 9.23 & 0.30 \\
\hline a039 & CLIQ study & 1543 & 17.8 & 200 & 20 & 3.65 & -3.68 & 1.84 & -1.79 & 3.63 & 0.06 \\
\hline $\mathrm{a} 041$ & Ramp rate & 17418 & 140.1 & 200 & 40 & 5.17 & -6.77 & 25.69 & -34.05 & 59.74 & 133.83 \\
\hline $\mathrm{a} 042$ & CLIQ study & 1544 & 17.8 & 200 & 10 & 3.02 & -3.16 & 1.51 & -1.56 & 3.07 & 0.06 \\
\hline $\mathrm{a} 043$ & CLIQ study & 3133 & 30.0 & 200 & 10 & 4.59 & -4.49 & 4.44 & -4.32 & 8.76 & 0.30 \\
\hline $\mathrm{a} 044$ & CLIQ study & 6652 & 57.1 & 200 & 10 & 5.36 & -5.48 & 13.40 & -13.71 & 27.11 & 7.74 \\
\hline $\mathrm{a} 045$ & Quench heater study & 16466 & 132.8 & - & - & - & - & - & & - & 120.43 \\
\hline $\mathrm{a} 046$ & Quench heater study & 16466 & 132.8 & - & - & - & - & - & & - & 120.43 \\
\hline $\mathrm{a} 047$ & Quench heater study & 16466 & 132.8 & - & - & - & - & - & & - & 120.43 \\
\hline $\mathrm{a} 048$ & Quench heater study & 1648 & 18.6 & - & - & - & - & - & & - & 1.33 \\
\hline a049 & Quench heater study & 16466 & 132.8 & - & - & - & - & - & & - & 120.43 \\
\hline a050 & Quench heater study & 3298 & 31.3 & - & - & - & - & - & & - & 5.38 \\
\hline $\mathrm{a} 051$ & Quench heater study & 8238 & 69.4 & - & - & - & - & - & & - & 31.83 \\
\hline $\mathrm{a} 052$ & Quench heater study & 13176 & 107.4 & - & - & - & - & - & & - & 79.57 \\
\hline $\mathrm{a} 053$ & Quench heater study & 1647 & 18.6 & - & - & - & - & - & & - & 1.33 \\
\hline $\mathrm{a} 054$ & Quench heater study & 3297 & 31.3 & - & - & - & - & - & & - & 5.38 \\
\hline $\mathrm{a} 055$ & Quench heater study & 8237 & 69.4 & - & - & - & - & - & & - & 31.83 \\
\hline
\end{tabular}

The values of all the strain gauges are shown before and after the test campaign at $1.8 \mathrm{~K}$ along with the effects of two representative CLIQ discharges: the first one is at $8.2 \mathrm{kA}$ (file name ID: a006) and the second one is at $18.2 \mathrm{kA}$ (file name ID: a008).

Both discharges are performed with a CLIQ unit set at $200 \mathrm{~V}$ that would imply $+17.5 \%$ of $d I_{\text {star }} / d t$ than at nominal voltage. However, $d I_{\text {star }} d d t$ turns out to be only $1.8 \%$ higher in comparison with nominal design.

All the measurements are shown together with $B \dot{B}$ derived from the magnetic measurements.

\section{A. General overview}

The strain gauge measurements before and after the 52 quenches at $1.8 \mathrm{~K}$ are reported in the bar chart of Fig. 19. Most of the gauges before and after the test campaign show the same strain, indicating that no plastic deformation occurred. However, from the readings of the seven gauges (M_F2_P1_A, M_F2_P1_T, M_F2_P2_A, M_F2_P3_T, M_F4_P1_A, B_F4_P1_A, B_F4_P1_T) it appears that some residual deformations took place. 


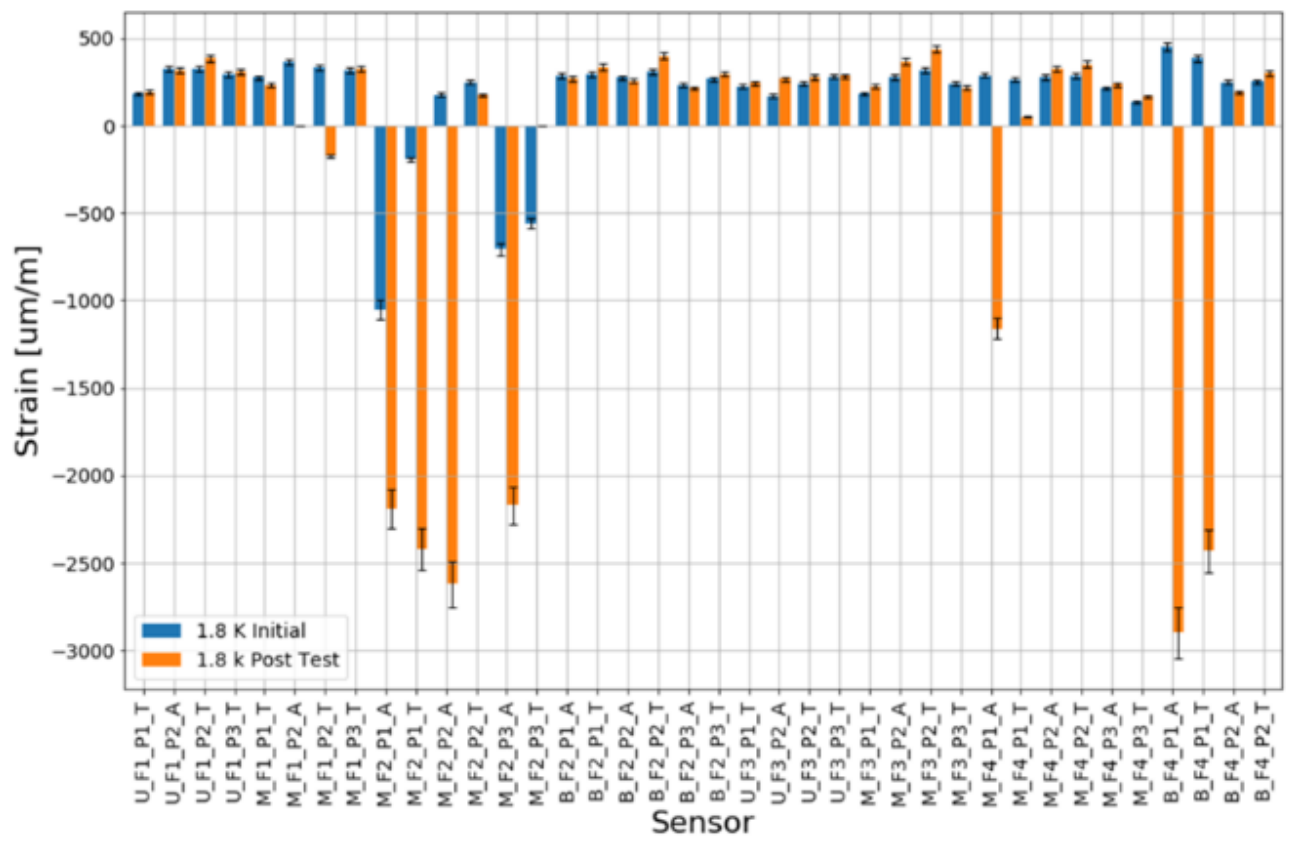

Fig. 19 Strain gauges signal before and after the 52 quenches at $1.8 \mathrm{~K}$. The error bar accounts for $5 \%$ of the measurement [35].

After a visual inspection of the beam screen, the strain gauges relative to the residual deformations were found either damaged or badly glued (see Fig. 20). The glue used to bound the gauge was left over the dedicated area creating a contact between the beam screen and the absorber The pressure and some movements of the heat absorbers might have been detected leading to these inaccurate results.

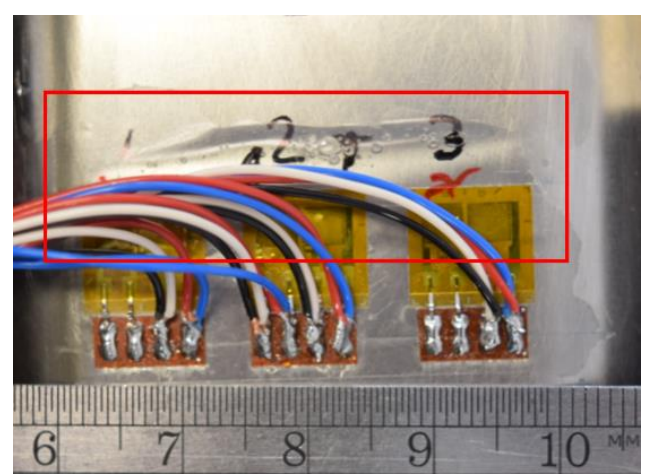

(a)

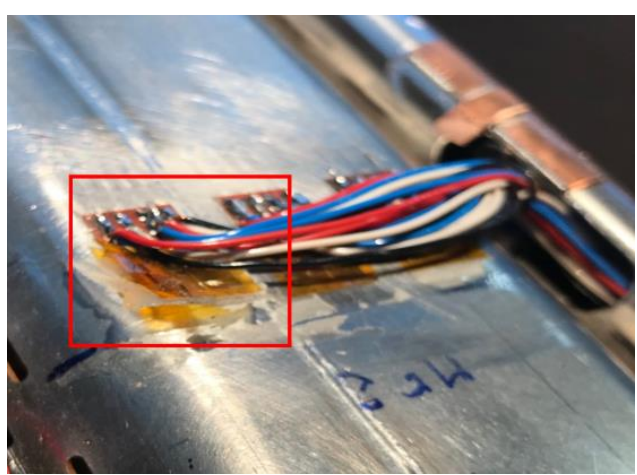

(b)

Fig. 20 Extra glue used to install some gauges in (a) and example of damage from tearing in (b) outlined by the red boxes.

\section{B. Measurements at $8.2 \mathrm{kA}$}

The transversal strains of the beam screen at $8.2 \mathrm{kA}$ are shown along with $B \dot{B}$ in Fig. 21 (a). As expected, the evolution in time of the strain is very similar to that of the magnetic measurement of Fig. 21 (c).

Some strain oscillations in the non-monotonic CLIQ discharge correspond to the oscillation of $B \dot{B}$ for the poles 1-3 and 2-4. In the monotonic phase, the highest value of strain corresponds to the highest absolute value of $B \dot{B}$. It is interesting to observe that the deflection in the magnetic measurement between $0.2 \mathrm{~s}$ and $0.4 \mathrm{~s}$, whose causes are unknown [22], is also present in the mechanical measurements. The mechanical and magnetic measurements are coherent further confirming the validity of the test campaign.

As the strain evolutions start from 0 and return to 0 the behavior of the beam screen can be considered fully elastic. The measured strain is not the same for all the faces of the beam screen. However, considering a Young's modulus of $200 \mathrm{GPa}$, the stress corresponding to a maximum measured strain of $220 \mu \mathrm{m} / \mathrm{m}$ is $44 \mathrm{MPa}$. These measurements are in agreement with simulations. 


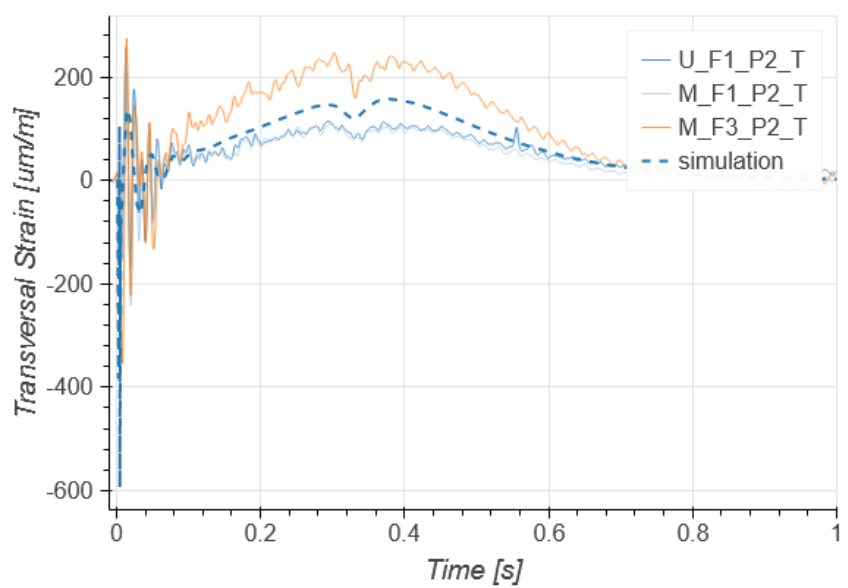

(a)

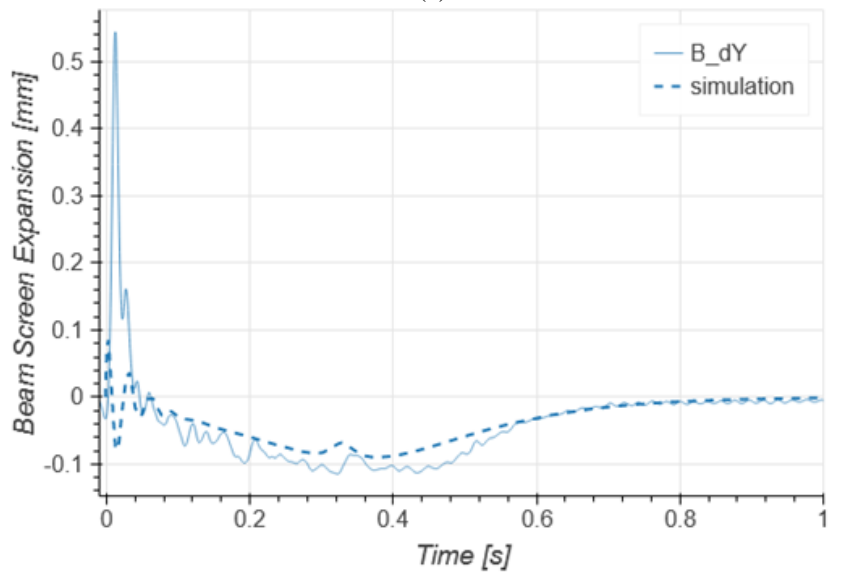

(b)

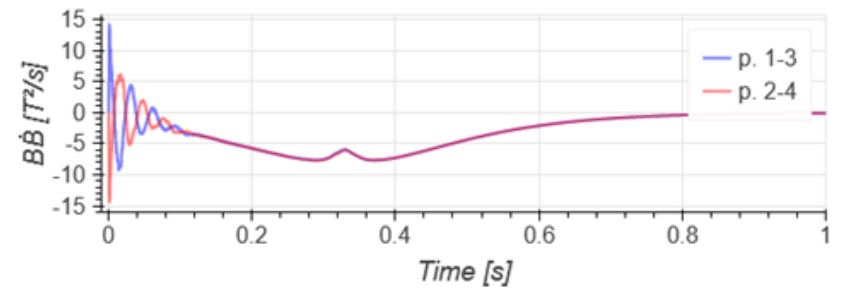

(c)

Fig. 21 In (a) transversal strains of the beam screen measured in the upper section of face 1-point 2 (U_F1_P2_T), in the middle section of face 1-point 2 (M_F1_P2_T), and in the middle section of face 3-point 2 (M_F3_P2_T) together with the relative simulated strain (simulation) at $8.2 \mathrm{kA}$. In (b) expansion of the beam screen measured by the bottom probe along the $\mathrm{y}$-axis $\left(\mathrm{B}_{-} \mathrm{dY}\right)$ together with the simulated one (simulation) at $8.2 \mathrm{kA}$. In (c) magnetic parameter, $B \dot{B}$, proportional to the induced forces, derived from the magnetic field measurements averaged for the couple of poles 1-3 (P. 1-3) and 2-4 (P. 2-4) at 8.2 kA.

The expansion of the beam screen measured by the single-pin-side of the probe is shown in Fig. 21 (b). A peak of around $0.5 \mathrm{~mm}$ appears at about $10 \mathrm{~ms}$. It is not deemed an actual peak as it was probably caused by the violent CLIQ dynamics perturbing the auto-equilibrated probe. A maximum expansion of $0.1 \mathrm{~mm}$ is recorded in the monotonic phase which is in agreement with the magnetic measurement and the simulations. 


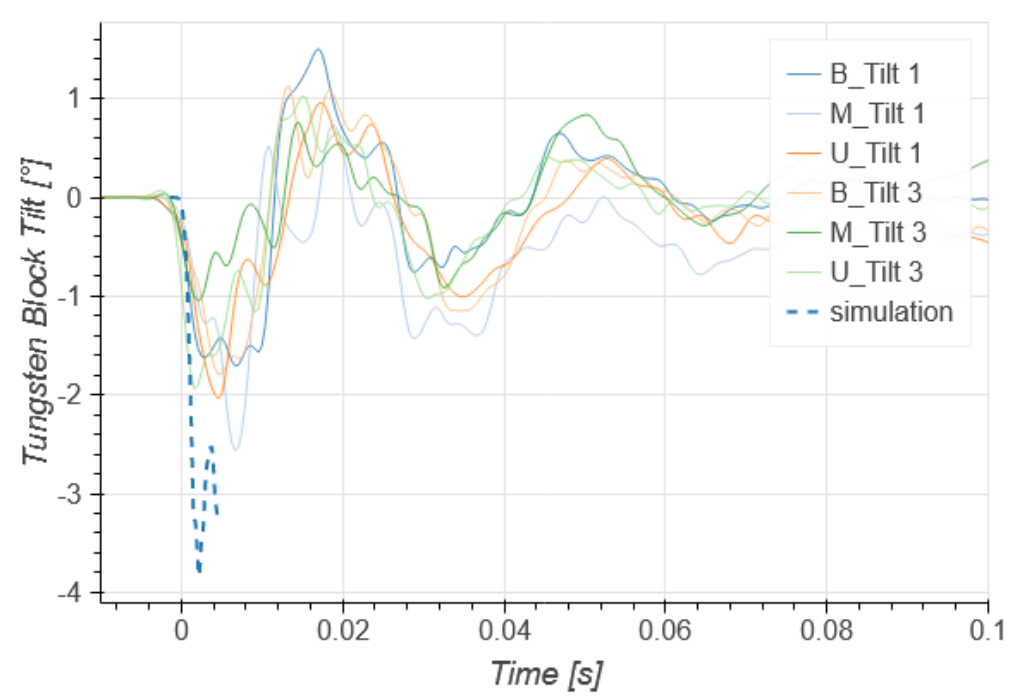

(a)

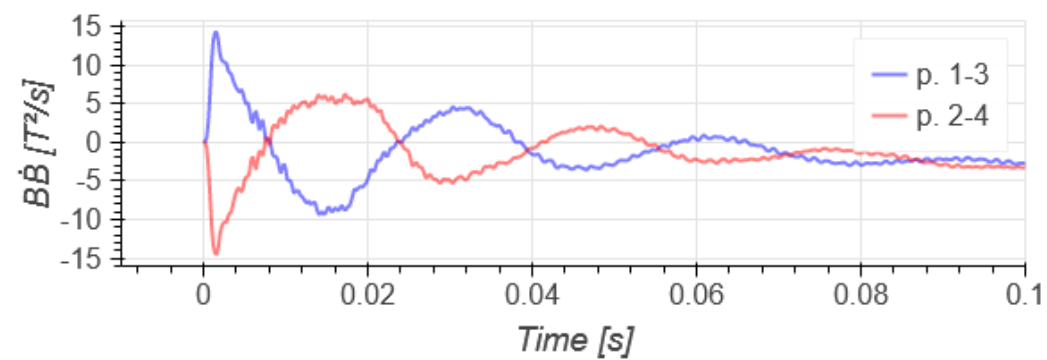

(b)

Fig. 22 In (a) tilt angles of the heat absorber measured in the bottom section of face 1 (B_Tilt 1), in the middle section of face 1 (M_Tilt 1), in the upper section of face 1 (U_Tilt 1), in the bottom section of face 3 (B_Tilt 3), in the middle section of face 3 (M_Tilt 3), in the upper section of face 3 (U_Tilt 3) together with the simulated tilt (simulation) at $8.2 \mathrm{kA}$. In (b) magnetic parameter, $B \dot{B}$, proportional to the induced forces, derived from the magnetic field measurements averaged for the couple of poles 1-3 (P. 1-3) and 2-4 (P. 2-4) at $8.2 \mathrm{kA}$.

Fig. 22 shows the tilt angle of the absorbers measured by the probe during the initial current oscillations of CLIQ. The tilt evolution of the absorber is in phase with the magnetic measurements. The difference between the local maxima and minima of $B \dot{B}$ of two consecutive poles matches in time the series of tilts.

The tilt changes direction as a function of $B \dot{B}$. In particular, when $B \dot{B}$ of the poles 1-3 is positive and that of the poles $2-4$ is negative, the direction is counterclockwise, whereas when $B \dot{B}$ of the poles 1-3 is negative and that of poles 2-4 is positive, the direction is clockwise. The highest tilt angle is around $2^{\circ}$ degrees counterclockwise; it occurs at around $5 \mathrm{~ms}$. The following tilts are less intense as the $B \dot{B}$ difference between consecutive poles decreases.

There is a factor 2 difference in comparison with simulation results. As shown by post-test simulation of Fig. 9, this might be related to the poor electrical contact between the absorber and the beam screen. This forces the current loop to partially close within the absorber itself and reduces, therefore, the induced forces.

\section{Measurements at $18.2 \mathrm{kA}$}

The transversal strains of the beam screen at $18.2 \mathrm{kA}$ is shown in Fig. 23 (a). Although the signal is not as clean as the one at $8.2 \mathrm{kA}$ some oscillations during the first part of the CLIQ discharge are still visible. The highest strain is around $1500 \mu \mathrm{m} / \mathrm{m}$ and occurs at the highest absolute value of $B \dot{B}$, namely $153 \mathrm{~T}^{2} / \mathrm{s}$ at around $60 \mathrm{~ms}$. Considering a Young's modulus of $200 \mathrm{GPa}$, the stress corresponding to the maximum strain is $300 \mathrm{MPa}$. The behavior of the beam screen is elastic as the strains return to 0 after the magnet is fully quenched. The time evolutions of the strains and of $B \dot{B}$, shown in Fig. 23 (c), are similar confirming the expected behaviour also at $18.2 \mathrm{kA}$. The comparison between measurements and simulations shows a good agreement and a $25 \%$ difference between peak values. The simulations turn out to be more conservative. 


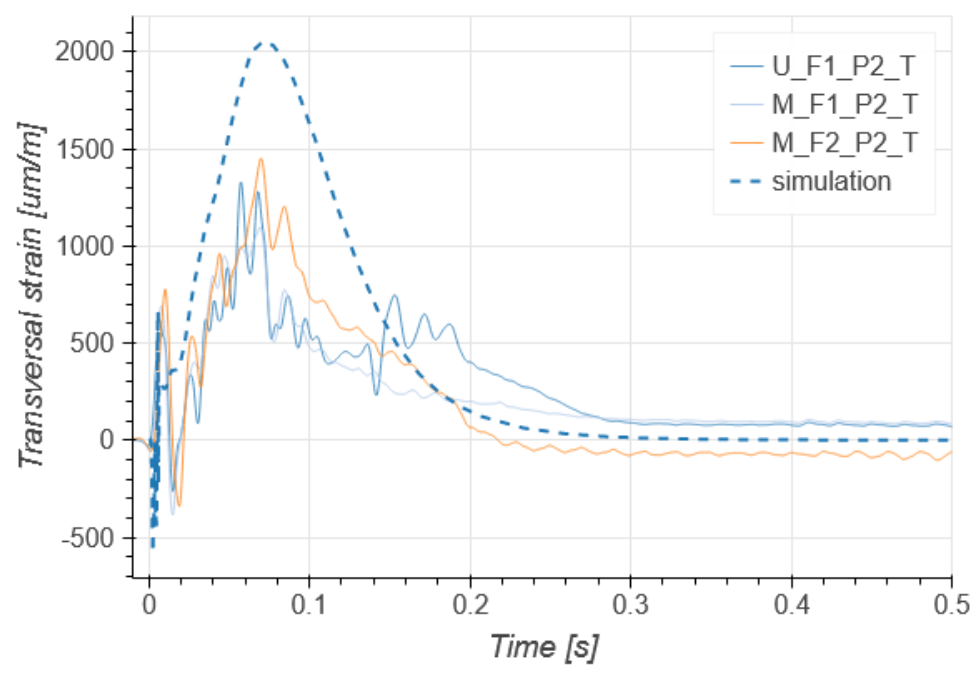

(a)

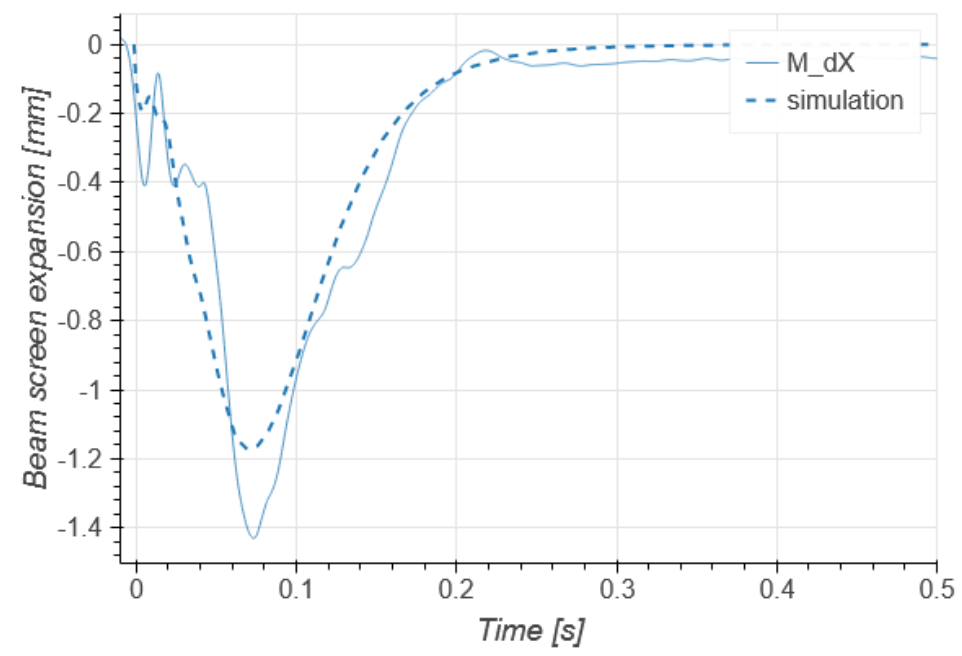

(b)

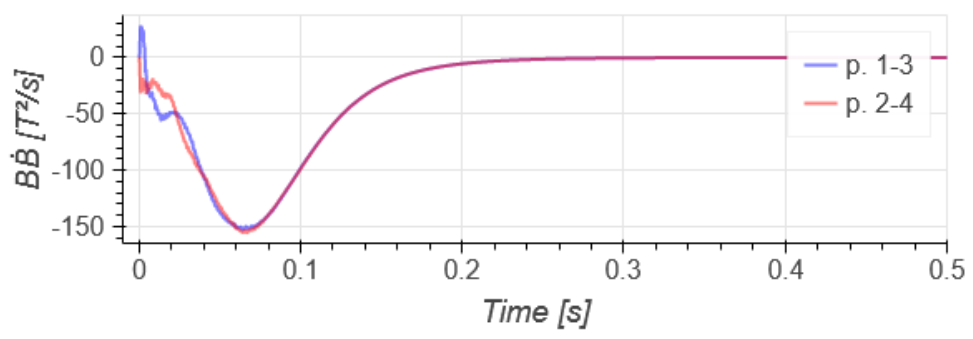

(c)

Fig. 23 In (a) transversal strains of the beam screen measured in the upper section of face 1-point 2 (U_F1_P2_T), in the middle section of face 1-point 2 (M_F1_P2_T), and in the middle section of face 2-point 2 (M_F2_P2_T) together with the relative simulated strain (simulation) at $18.2 \mathrm{kA}$. In (b) expansion of the beam screen measured by the middle probe along the $\mathrm{x}$-axis (M_dX) together with the simulated one (simulation) at $18.2 \mathrm{kA}$. In (c) magnetic parameter, $B \dot{B}$, proportional to the induced forces, derived from the magnetic field measurements averaged for the couple of poles 1-3 (P. 1-3) and 2-4 (P. 2-4) at 18.2 kA.

The expansion of the beam screen measured by the single-pin-side of the probe is shown in Fig. 23 (b). The maximum expansion is $1.4 \mathrm{~mm}$ and is in phase with magnetic measurement and simulation.

The tilt measured by the double-pin-side of the probe is not clear (see Fig. 24). The strong dynamics of the CLIQ discharge might lead the probe to resonate giving faulty results. 


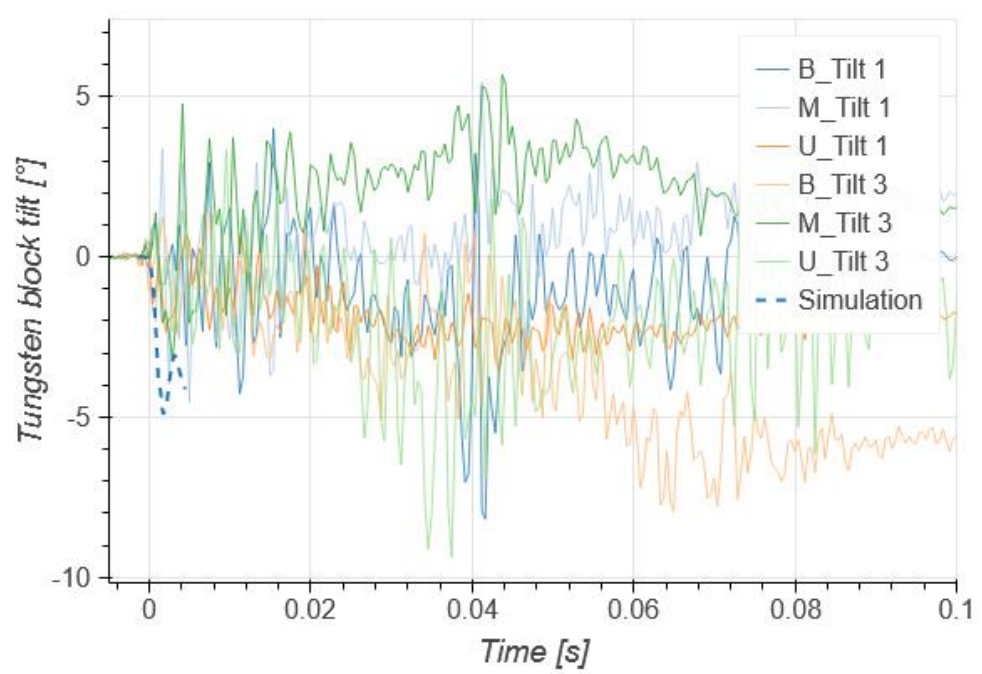

(a)

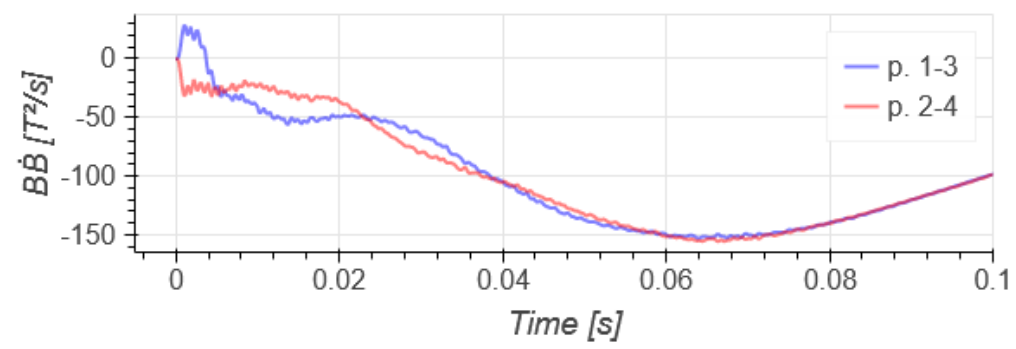

(b)

Fig. 24 In (a) tilt angles of the heat absorber measured in the bottom section of face 1 (B_Tilt 1), in the middle section of face 1 (M_Tilt 1), in the upper section of face 1 (U_Tilt 1), in the bottom section of face 3 (B_Tilt 3), in the middle section of face 3 (M_Tilt 3), and in the upper section of face 3 (U_Tilt 3 ) together with the simulated tilt (simulation) at $18.2 \mathrm{kA}$. In (b) magnetic parameter, $B \dot{B}$, proportional to the induced forces, derived from the magnetic field measurements averaged for the couple of poles 1-3 (P. 1-3) and 2-4 (P. 2-4) at $8.2 \mathrm{kA}$.

\section{Cold bore strains}

The transversal strains measured by the optical fibers mounted on the cold bore are shown at $13.2 \mathrm{kA}$ (file name: a007), 16.2 kA (file name: a020), and 18.2 kA (file name: a008) in Fig. 25-27, respectively. Only one fiber survived the cool down at $1.9 \mathrm{~K}$. The results are those of three measuring areas along the generatrix at $0^{\circ}$ degree angle. As for the beam screen, the strain follows the time evolution of $B \dot{B}$. Some strain oscillations are present during the CLIQ phase which are lower than the strain reached during the monotonic phase. The highest transversal strains are $30 \mu \mathrm{m} / \mathrm{m}$ at $13.2 \mathrm{kA}, 80 \mu \mathrm{m} / \mathrm{m}$ at $16.2 \mathrm{kA}$ and $130 \mu \mathrm{m} / \mathrm{m}$ at $18.2 \mathrm{kA}$. This latter strain corresponds to $26 \mathrm{MPa}$. A contact between the absorber and the cold bore is assumed due to the strains in phase with the oscillations of the CLIQ discharge. In fact, from simulation, the maximum strains of the cold bore would be smaller by about a factor of 2 if the heat absorbers were not considered, i.e. without any dynamic contact. However, the maximum strain of the cold bore calculated for the nominal design is a factor 4 - 5 lower. As shown in Fig. 9, it is believed that this is due to the poor electrical contact between the absorber and the octagonal tube impacting also the forces induced in the absorbers which turn out to be milder than expected (see Fig. 22).

The HL-LHC beam screen is by concept a very flexible assembly and it was designed to behave elastically during a quench. For this purpose, the absorbers are not welded on the octagonal tube but are retained by elastic titanium rings around the tube.

During a quench, the forces induced in the beam screen are transferred to the cold bore with no plastic deformations.

After a quench, the absorbers can return to their nominal position thanks to the elastic rings. Hence, no thermal contact with the cold bore is expected. 
13.2 kA

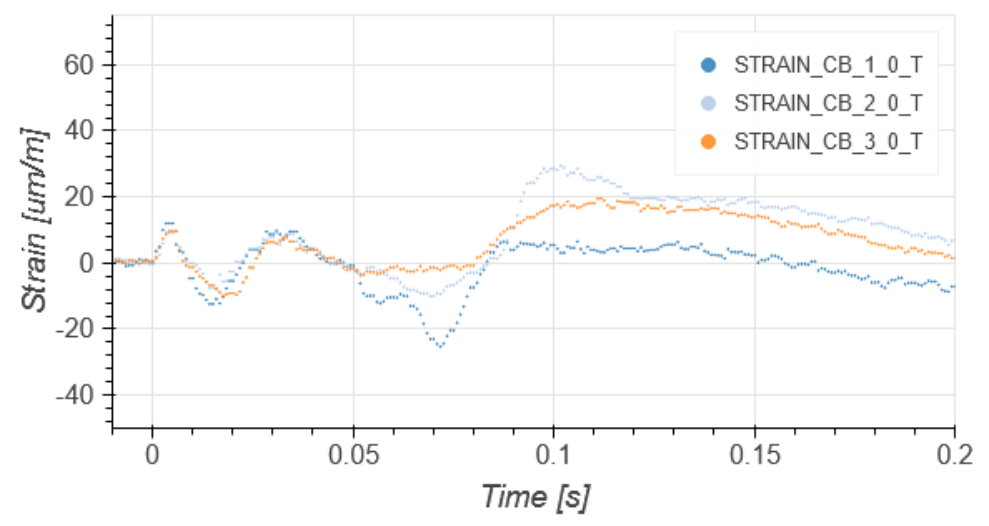

(a)

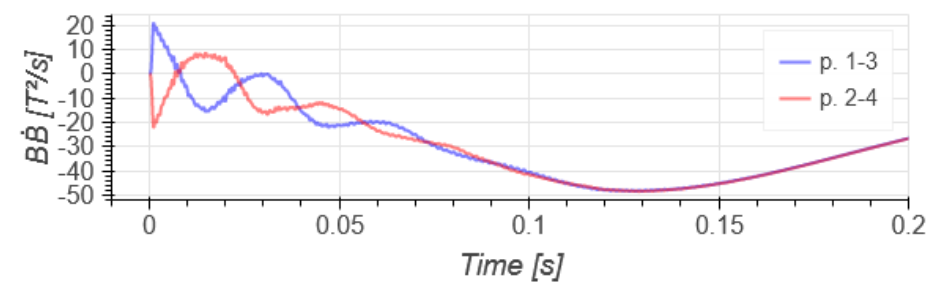

(b)

Fig. 25 In (a) transversal strains of the cold bore measured in the zone 1 at $0^{\circ}$ (STRAIN_CB_1_0_T), in the zone 2 at $0^{\circ}$ (STRAIN_CB_2_0_T), and in the zone 3 at $0^{\circ}$ (STRAIN_CB_3_0_T) at $13.2 \mathrm{kA}$. In (b) magnetic parameter, $B \dot{B}$, proportional to the induced forces, derived from the magnetic field measurements averaged for the couple of poles 1-3 (P. 1-3) and 2-4 (P. 2-4) at $13.2 \mathrm{kA}$.

$16.5 \mathrm{kA}$

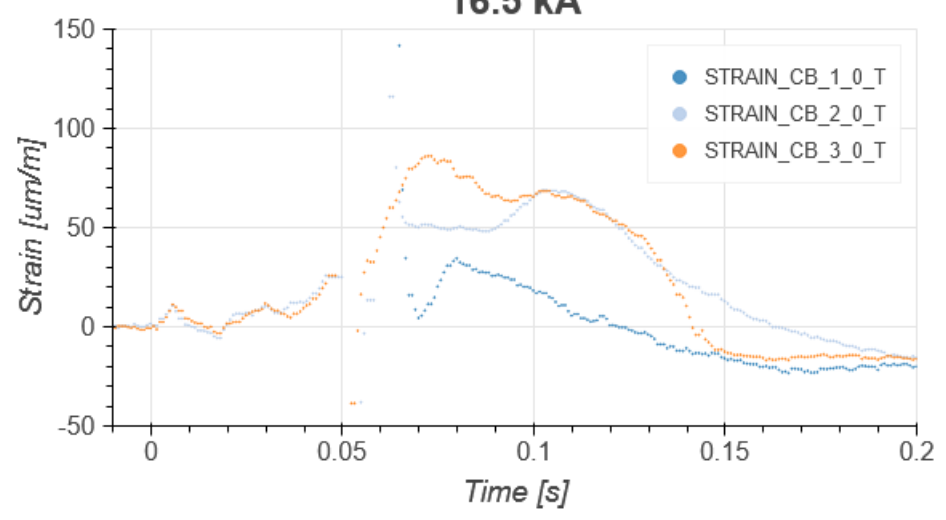

(a) 


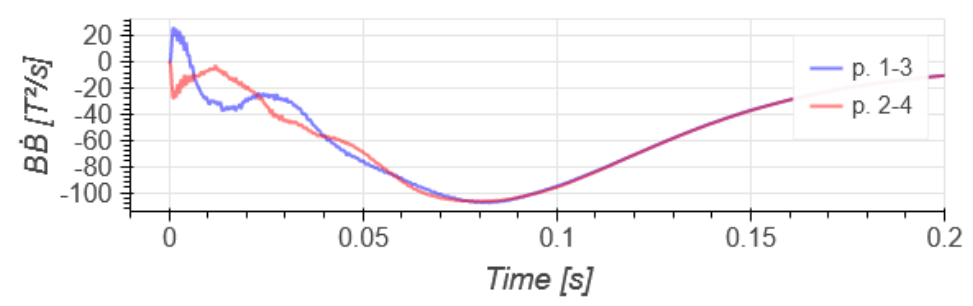

(b)

Fig. 26 In (a) transversal strains of the cold bore measured in the zone 1 at $0^{\circ}$ (STRAIN_CB_1_0_T), in the zone 2 at $0^{\circ}$ (STRAIN_CB_2_0_T), and in the zone 3 at $0^{\circ}$ (STRAIN_CB_3_0_T) at $16.5 \mathrm{kA}$. In (b) magnetic parameter, $B \dot{B}$, proportional to the induced forces, derived from the magnetic field measurements averaged for the couple of poles 1-3 (P. 1-3) and 2-4 (P. 2-4) at $16.5 \mathrm{kA}$.

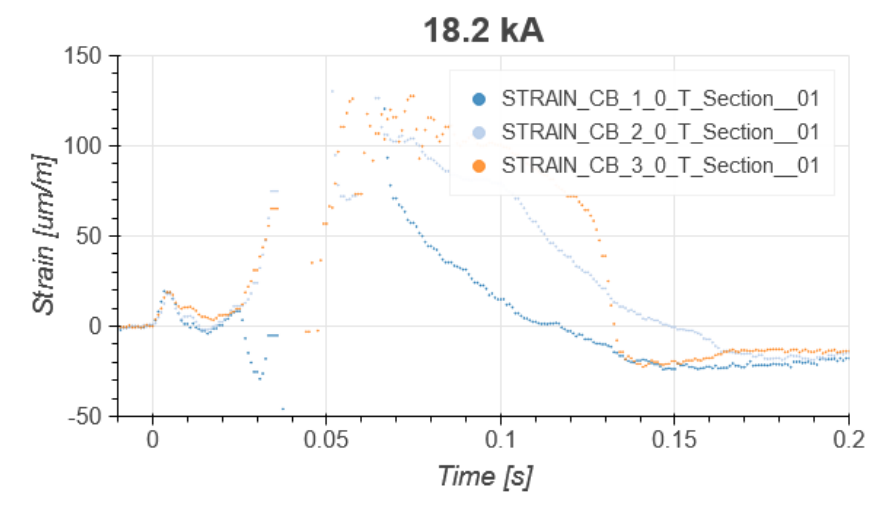

(a)

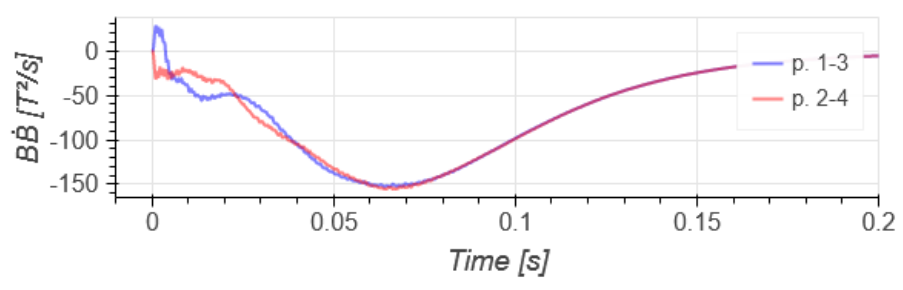

(b)

Fig. In (a) transversal strains of the cold bore measured in the zone 1 at $0^{\circ}$ (STRAIN_CB_1_0_T), in the zone 2 at $0^{\circ}$ (STRAIN_CB_2_0_T), and in the zone 3 at $0^{\circ}$ (STRAIN_CB_3_0_T) at $18.2 \mathrm{kA}$. In (b) magnetic parameter, $B \dot{B}$, proportional to the induced forces, derived from the magnetic field measurements averaged for the couple of poles 1-3 (P. 1-3) and 2-4 (P. 2-4) at 18.2 kA.

\section{E. Thermal link behavior}

The behavior of the thermal links during a magnet quench is not the same for all of them. Although a periodicity was observed in the links of each block, the external ones get slightly deformed towards the outside while the central ones remain almost in their original shape (see Fig. 28).

As shown by simulation in Fig. 9, the forces of the external links are driven by the current induced in the absorber, which flows in such links to form a loop. On the contrary, independent current loops are induced in the central links giving rise to opposite direction forces, thus, limiting the deformation of the link.

The deformations of the thermal links are not excessive and can be tolerated. No damage to the welds of the flexible part of the link was observed. The tested design corresponds to a worst-case scenario as the full thermal link was initially deformed toward the cold bore. The nominal shape is designed to be much less sensitive to the phenomenon and the observed deformation after the quench, even if not critical, is not expected. In addition, the number of layers of the flexible segments is reduced from 10 to 8 . The thickness of the first and last layers is increased from 0.1 to $0.2 \mathrm{~mm}$ resulting in a more robust structure. 


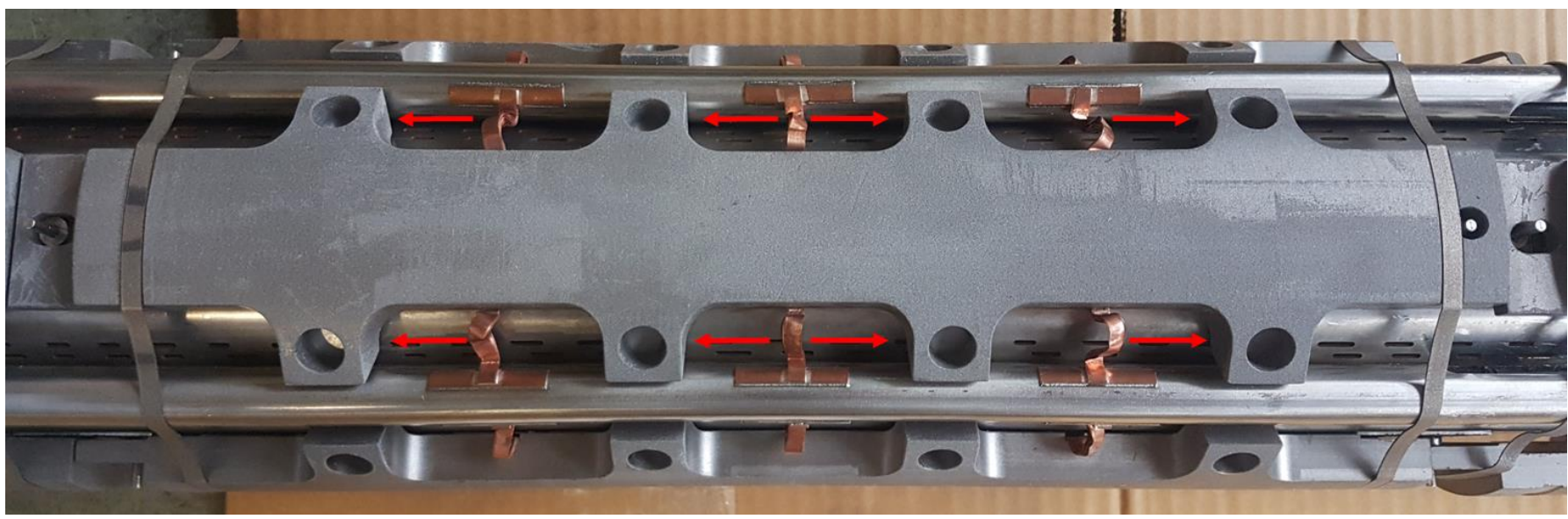

Fig. 28 Deformations of the thermal links of one heat absorber after 52 quenches. The distribution of the total forces is represented by red arrows.

\section{CONCLUSIONS}

The mechanical integrity of the Q1-type beam screen prototype was demonstrated. The behavior of the beam screen remains elastic after 52 quenches up to a current of $18.2 \mathrm{kA}$ (the ultimate operational current is $17.8 \mathrm{kA}$ ).

The thermal links, the elastic rings and the centering pins were visually inspected after the test and no unexpected deformation was observed. During a quench, the beam screen behaves as expected. First, the torque induced during the first phase of CLIQ was observed. Second, the heat absorbers go into contact with the cold bore and return to their original position.

The mechanical response of the beam screen is directly correlated with the magnetic field measurements and driven by $B \dot{B}$.

The transversal strain of the beam screen is around $25 \%$ lower than expected from simulation at $18.2 \mathrm{kA}$.

The behavior of the beam screen was predicted by simulations with acceptable approximation. Hence, the measurements assure the validity of the design of the HL-LHC beam screen.

\section{ACKNOWLEDGMENT}

Research supported by the HL-LHC project.

The authors would like to warmly thank the CERN colleagues involved in the test and in particular: P. Ferracin, E. Todesco, J.C. Perez, S. Izquierdo Bermudez, N. Bourcey, E. Ravaioli, M.Bajko and F.J. Mangiarotti.

\section{REFERENCES}

[1] E. Todesco, H. Allain, G. Ambrosio, G. Arduini, F. Cerutti, R. De Maria, L. Esposito, S. Fartoukh, P. Ferracin, H. Felice, R. Gupta, R. Kersevan, N. Mokhov, T. Nakamoto, I. Rakno, J. M. Rifflet, L. Rossi, G. L. Sabbi, M. Segreti, F. Toral, Q. Xu, P. Wanderer, and R. van Weelderen, "A first baseline for the magnets in the high luminosity lhe insertion regions," IEEE Transactions on Applied Superconductivity, vol. 24, no. 3, pp. 1-5, Jun. 2014.

[2] G. Apollinari, I. Bejar Alonso, O. Brüning, P. Fessia, M. Lamont, L. Rossi, and L. Tavian, (editors) "High-Luminosity Large Hadron Collider (HL-LHC) Technical Design Report V.0.1," CERN, Geneva, Switzerland, Yellow Reports: Monographs., vol. 4, 2017. [Online]. Available: http://dx.doi.org/10.23731/CYRM-2017-004. 
[3] E, Métral, F. Caspers, N. Mounet, T. Pieloni and B. Salvant, "Impedance and Component Heating. In The High Luminosity Large Hadron Collider: The New Machine for Illuminating the Mysteries of Universe,” Adv. Ser. Dir. High Energy Phys., vol. 24, pp. 269-280, 2015. Available: https://doi.org/10.1142/9789814675475_0015.

[4] G. Skripka and G. Iadarola, "Electron cloud build-up in two-beam regions for HL-LHC, heat load and vacuum aspects", CERN, Geneva, Switzerland, Yellow Rep. Conf. Proc., vol. 7, pp. 59-64, 2020.

[5] V. Baglin, and C. Garion, "Conceptual Specification HL-LHC shielded beam screen [LHC-VSM]," Geneva, Switzerland, Tech. Rep. EDMS 1361079, 2014.

[6] K. Artoos, A. Jacquemod, A. Poncet, and R. J. M. Veness, "Mechanical and Thermal Measurements on a 1 m Long Model Beam Screen (With Slots) during Quenches”, CERN, Geneva, Switzerland, Tech. Rep. LHC-Project-Note-35, 1996.

[7] K. Artoos, P. Cruikshank, and N. Kos, "Mechanical and thermal measurements on an $11 \mathrm{~m}$ long beam screen in the LHC Magnet Test String during RUN 3A”, CERN, Geneva, Switzerland, Tech. Rep. LHC-Project-Note-178, 1999.

[8] C. Rathjen, "First quench tests of a prototype LHC racetrack type beam screen in a short dipole magnet model," Tech. Note CERN EST-ESI/99-13.

[9] K. Artoos, J. M-Darve, and C. Rathjen. "Beam screen quench test in a 15 metre long LHC dipole magnet", CERN, Geneva, Switzerland, Tech. Rep. EST-ME/2001-003, 2001.

[10] S. Sgobba, "Materials for high vacuum technology, an overview," CERN, Geneva, Switzerland, Tech. Rep., 2006.

[11] P. C. Pinto, S. Calatroni, H. Neupert, D. Letant-Delrieux, P. Edwards, P. Chiggiato and S. Lucas, "Carbon coatings with low secondary electron yield", Vacuum, 98, 29-36, 2013.

[12] R. Kersevan, C. Garion, and N. Kos, "Preliminary Design of the HiLumi-LHC Triplet Area Beam Screen," CERN, Geneva, Switzerland, CERN-ACC-2014-0268, 2014.

[13] C. Garion, V. Baglin, and R. Kersevan, "Preliminary Design of the High-Luminosity LHC Beam Screen with Shielding," CERN, Geneva, Switzerland, CERN-ACC-2015-352, 2015.

[14] V. Baglin, L. Tavian, P. Lebrun, and R. van Weelderen, "Cryogenic beam screens for high- energy particle accelerators," CERN, Geneva, Switzerland, CERN-ATS-2013-006, 2013.

[15] Morrone, M., C. Garion, M. Aurisicchio, and P. Chiggiato, “A coupled multiphysics fem model to investigate electromagnetic, thermal and mechanical effects in complex assemblies: The design of the high-luminosity large hadron collider beam screen," Applied mathematical modelling, vol. 57, pp.280-301, May. 2018.

[16] M. Morrone, "Thermomechanical study of complex structures in the aperture of superconducting magnets: application to the design of the High-Luminosity LHC shielded beam screen”, Doctoral dissertation, Imperial College London, 2018, Available: https://ethos.bl.uk/OrderDetails.do?uin=uk.bl.ethos.745332.

[17] C. Rathjen, "Mechanical behaviour of vacuum chambers and beam screens under quench conditions in dipole and quadrupole fields," CERN, Geneva, Switzerland, Tech. Rep. No. LHC-Project-Report-582, 2002.

[18] R. Valbuena, "Calcul des Courants de Foucault et Analyse Mecanique de l'ecran de Faisceau des Quadrupoles $<$ Low-Beta > Supraconducteurs du LHC en cas de Transition Resistive," CERN, Geneva, Switzerland, Tech. Rep., 2002.

[19] E. Ravaioli, “CLIQ. A new quench protection technology for superconducting magnets," Ph.D. dissertation, University of Twente, Enschede, NL, 2015.

[20] M. Morrone, "Mechanical effects of the HL-LHC beam screen misalignment within the cold bore," Geneva, Switzerland, Tech. Rep. EDMS 1966571, 2006.

[21] C. Garion, "Status of beam screen / cold bore / interconnect / CWT design \& production", Talk given at the $8^{\text {th }}$ HL-LHC Collaboration Meeting in Geneva, 2018. [Online]. Available: https://indico.cern.ch/event/742082/contributions/3085182/ 
[22] E. Ravaioli, personal communication by email, 14 September 2018.

[23] E. Ravaioli, V. L. Datskov, C. Giloux, G. Kirby, H. H. ten Kate, and A. P. Verweij, "New, coupling loss induced, quench protection system for superconducting accelerator magnets, " IEEE transactions on applied superconductivity, vol. 24(3), pp. 15, Sep. 2013.

[24] S. Sgobba, and G. Hochortler, "New non-magnetic stainless steel for very low temperature applications," in International Congress Stainless Steel'99 Science and Market 3 rd European Congress Proceedings, Chia Laguna, Italy, 1999, Vol. 2, pp. 391-401.

[25] K. Suzuki, J. Fukakura, and H. Kashiwaya, "Cryogenic fatigue properties of 304L and 316L stainless steels compared to mechanical strength and increasing magnetic permeability, "Journal of testing and evaluation, 16(2), 190-197, Mar. 1988.

[26] E. S. Drexler, N. J. Simon, and R. P. Reed, "Properties of copper and copper alloys at cryogenic temperatures," Boulder, CO, USA, Tech. Rep. NIST Monograph 177, 1992. [Online]. Available: https://nvlpubs.nist.gov/nistpubs/Legacy/MONO/nistmonograph177.pdf

[27] A. F. Clark, G. E. Childs, and G. H. Wallace, "Electrical resistivity of some engineering alloys at low temperatures," Cryogenics, vol. 10, no. 4, pp. 295-305, Aug. 1970.

[28] C. Garion, L. Dufay-Chanat, T. Koettig, W. Machiocha, and M. Morrone, "Material characterisation and preliminary mechanical design for the HL-LHC shielded beam screens operating at cryogenic temperatures, " In IOP Conference Series: Materials Science and Engineering, vol. 102, no. 1, p. 012013. IOP Publishing, 2015.

[29] M. Morrone, "Electrical resistivity and thickness measurements of electroplated copper for the quench test of the Q1-type HL-LHC shielded beam screen,” Geneva, Switzerland, Tech. Rep. EDMS 2256209, 2019.

[30] Standard Guide for Installing Bonded Resistance Strain Gages, ASTM E1237-93, West Conshohocken, PA, 2014.

[31] Standard Practice for Calibration of Linear Displacement Sensor Systems Used to Measure Micromotion, ASTM F2537-06, 2017.

[32] G. Morgan, "Stationary coil for measuring the harmonics in pulsed transport magnets," in Proceedings of the Fourth International Conference on Magnet Technology, Brookhaven National Lab., Upton, NY, USA, 1972, pp. 787-790.

[33] L. Walckiers, "Magnetic measurement with coils and wires," CERN, Geneva, Switzerland, arXiv:1104.3784, 2011.

[34] M. Guinchard, A. Bertarelli, L. Bianchi, F. Boyer, M. Cabon, M. Calviani and P. Grosclaude, "Mechanical Strain Measurements Based on Fiber Bragg Grating Down to Cryogenic Temperature-R\&D Study and Applications," 9th Int. Particle Accelerator Conf. IPAC'18, Vancouver, BC, Canada, pp. 2572-2574, 2018.

[35] A. Bouchardy, "Caractérisation des mesures de déformation par jauges d'extensomètrie à température cryogénique," Geneva, Switzerland, Tech. Rep. EDMS 1073153, 2010. 